\title{
FRAGMENTS OF ARITHMETIC AND TRUE SENTENCES
}

\author{
A. Cordón-Franco, A. Fernández-Margarit, and F.F. Lara-Martín \\ Dpto. Ciencias de la Computación e Inteligencia Artificial \\ Facultad de Matemáticas. Universidad de Sevilla \\ C/ Tarfia, s/n, 41012 Sevilla (Spain) \\ \{acordon, afmargarit, fflara\}@us.es
}

\begin{abstract}
By a theorem of R. Kaye, J. Paris and C. Dimitracopoulos, the class of the $\Pi_{n+1}$-sentences true in the standard model is the only (up to deductive equivalence) consistent $\Pi_{n+1}$-theory which extends the scheme of induction for parameter free $\Pi_{n+1}$-formulas. Motivated by this result, we present a systematic study of extensions of bounded quantifier complexity of fragments of first-order Peano Arithmetic. Here, we improve that result and show that this property describes a general phenomenon valid for parameter free schemes. As a consequence, we obtain results on the quantifier complexity, (non)finite axiomatizability and relative strength of schemes for $\Delta_{n+1}$-formulas.
\end{abstract}

\section{Introduction}

In this paper we shall deal with parameter free fragments of Arithmetic, that is, those subsystems of first-order Peano Arithmetic obtained by restricting some axiom scheme (induction, minimization and collection) to a class of formulas without parameters. A central paper on this topic is [15], where R. Kaye, J. Paris and C. Dimitracopoulos introduced parameter free fragments for $\Sigma_{n}$ and $\Pi_{n}$ formulas and proved their basic properties. This work was further developed by Kaye [13], Z. Adamowicz and T. Bigorajska [1, 5], L. Beklemishev [3, 4], and others, pointing out tight relationships among parameter free schemes, classification of provably total recursive functions and subsystems of Arithmetic described in terms of inference rules or reflection principles. All these works provide evidence that the behaviour of parameter free fragments is very different from their parameter counterparts.

The aim of this paper is to investigate one of those differences: the existence of extensions with small quantifier complexity. In [19] A. Wilkie proved that the scheme of induction for parameter free universal formulas $\mathbf{I} \forall_{1}^{-}$does not have a universal axiomatization but does have a universal extension: $\mathbf{T h}_{\forall_{1}}(\mathcal{N})$ (the theory of the $\forall_{1}$-sentences true in the standard model of Arithmetic). To establish this result it is shown that $\mathbf{T h}_{\forall_{1}}(\mathcal{N})$ is the only (up to deductive equivalence) consistent $\forall_{1}$-theory which extends $\mathbf{I} \forall_{1}^{-}$and Wilkie asked if the same was true for $\mathbf{I} \forall_{n+1}^{-}$and $\mathbf{T h}_{\forall_{n+1}}(\mathcal{N})$ for $n \geq 1$. In his thesis [13] Kaye gave a positive answer to Wilkie's

Partially supported by the Andalusian Government, grant TIC-137. 
question. By Kaye's analysis of Matiyasevič's theorem on the diophantine representation of recursively enumerable predicates, it follows that, for $n \geq 1$, Matiyasevič's theorem is provable both in $\mathbf{T} \mathbf{h}_{\forall_{n+1}}(\mathcal{N})$ and in $\mathbf{I} \forall_{n+1}^{-}$. So, for $n \geq 1, \mathbf{T h}_{\forall_{n+1}}(\mathcal{N})$ and $\mathbf{T h}_{\Pi_{n+1}}(\mathcal{N})$ are deductively equivalent and $\mathbf{I} \forall_{n+1}^{-}$is equivalent to the parameter free $\Pi_{n+1}$-induction scheme $\mathbf{I} \Pi_{n+1}^{-}$. Then Wilkie's question is answered by proving that $\mathbf{T} \mathbf{h}_{\Pi_{n+1}}(\mathcal{N})$ is the only (up to deductive equivalence) consistent $\Pi_{n+1}$-theory which implies $\mathbf{I} \Pi_{n+1}^{-}$. The fragment $\mathbf{I} \Pi_{n+1}^{-}$is $\Sigma_{n+2}$-axiomatized but not $\Pi_{n+2}$. Therefore, for each $n, \mathbf{I} \Pi_{n+1}^{-}$has consistent extensions of quantifier complexity less than $\Sigma_{n+2}$ (the one given by its natural formulation), but necessarily of big descriptive complexity (such extensions correspond to $\Pi_{n+1}^{0}$-complete sets).

Our purpose is to show that this result is not a particular property of $\mathbf{I} \Pi_{n+1}^{-}$, but actually a general phenomenon valid for parameter free schemes. More concretely, we deal with the following problem:

Given a fragment of Arithmetic, T,

- determine the least level $\Gamma$ in the classical $\Sigma_{n} / \Pi_{n}$ Arithmetic Hierarchy such that there exists some consistent and $\Gamma$-axiomatized theory which extends $\mathbf{T}$

- in the case that $\mathbf{T}$ has consistent extensions of quantifier complexity less than that of its axiomatization, determine the descriptive complexity of such extensions.

In order to describe in a simple way the results obtained in this work, we introduce the following measures for the complexity of extensions of $\mathbf{T}$ (where $\mathbf{T h}_{\Pi_{m}}(\mathbf{T})$ denotes the class of the $\Pi_{m}$-sentences provable in $\mathbf{T}$ ):

(1) (Syntactical complexity) We say that $\mathbf{T}$ has $\Gamma$-extensions if there is some consistent and $\Gamma$-axiomatized theory which implies $\mathbf{T}$.

(2) (Descriptive complexity) Let $k, m \geq 1$. Assume that $\mathbf{T}$ has $\Pi_{k}$-extensions.

(a) We say that $\mathbf{T}$ is of type $k \rightarrow m$ if for each $\Pi_{k}$-extension of $\mathbf{T}, \mathbf{T}^{\prime}$, it holds that $\mathbf{T h}_{\Pi_{m}}\left(\mathbf{T}^{\prime}\right)=\mathbf{T} \mathbf{h}_{\Pi_{m}}(\mathcal{N})$.

(b) We say that $\mathbf{T}$ is of type $k \stackrel{w}{\longrightarrow} m$ if for each $\Pi_{k}$-extension of $\mathbf{T}, \mathbf{T}^{\prime}$, it holds that $\mathbf{T} \mathbf{h}_{\Pi_{m}}(\mathcal{N})$ is recursive in $\mathbf{T} \mathbf{h}_{\Pi_{m}}\left(\mathbf{T}^{\prime}\right)$.

Notice that we can reformulate Kaye-Paris-Dimitracopoulos' result on $\mathbf{I \Pi}_{n+1}^{-}$by saying that this theory is of type $n+1 \rightarrow n+1$.

Our main motivation for a systematic analysis of properties of extensions of fragments of Arithmetic is to use these properties in order to obtain (usual) results on fragments of Arithmetic such as quantifier complexity, (non)finite axiomatizability or relative strength. For instance, let us observe that for a theory $\mathbf{T}$ to be of type $k \rightarrow m$ is a strong way of saying $\mathbf{T}$ is not finitely axiomatized. Moreover, the investigation of these properties of fragments of Arithmetic naturally leads to new conditions to solve some open problems in this area.

In [10] some previous work in this direction was developed in connection with (the parameter free version of) Paris-Friedman's problem on the equivalence between the schemes of induction and minimization for $\Delta_{n+1}$-formulas. The present paper can be considered to be an extension of [10]. We improve some results and give partial answers to some open problems there. Besides we simplify some proofs, especially 
for results on $\Delta_{n+1}$-induction scheme, both for its parameter free version, $\mathbf{I} \Delta_{n+1}^{-}$, and for its uniform version, UI $\Delta_{n+1}$ (see section 2 for a detailed formulation of these fragments). Proofs given in [10] for determining the quantifier complexity and (non)finite axiomatizability of theories $\mathbf{I} \Delta_{n+1}^{-}$, $\mathbf{U I} \Delta_{n+1}$ make use of the Arithmetized Completeness Theorem. On the other hand, as remarked by the anonymous referee, some results obtained in this paper can be also proved using reflection principles (see $[3,4,16,20]$ ). Here we offer alternative proofs which employ a basic technique in the study of fragments of Arithmetic; namely, the construction of submodels using definable elements.

To close this section we describe and briefly discuss the main results obtained in this paper. In section 3 we refine basic results on models constructed using definable elements. In section 4 we use those results to develop a systematic study of the syntactical and descriptive complexity of extensions of fragments. Diagrams in Theorem 1.1 summarize our main results on extensions of fragments (let us remark that $\bigvee_{n}$ denotes the class of formulas $\left\{\varphi \vee \theta: \varphi \in \Sigma_{n}, \theta \in \Pi_{n}\right\}$ and $\cup_{n}$ denotes the class $\left.\Sigma_{n} \cup \Pi_{n}\right)$.

\section{Theorem 1.1.}

- (Syntactical complexity):

\begin{tabular}{|c|c|c|c|}
\hline Theories & $\begin{array}{c}\mathbf{I} \Sigma_{n+1}, \mathbf{B} \Sigma_{n+1} \\
\mathbf{I} \Delta_{n+1}\end{array}$ & $\begin{array}{l}\mathbf{I} \Sigma_{n+1}^{-}, \mathbf{B} \Sigma_{n+1}^{-} \\
\quad \mathbf{U I} \Delta_{n+1}\end{array}$ & $\begin{array}{c}\mathbf{I} \mathbf{I}_{n+1}^{-} \\
\mathbf{L} \Delta_{n+1}^{-}, \mathbf{I} \Delta_{n+1}^{-}\end{array}$ \\
\hline Quantifier complexity & $\Pi_{n+3}$ & $\bigvee_{n+2}$ & $\Sigma_{n+2}$ \\
\hline$\Gamma$-extensions & $\begin{array}{l}\Pi_{n+3} \\
\text { but not }{ }^{(1)} \Sigma_{n+3}\end{array}$ & $\begin{array}{l}\Pi_{n+2} \\
\text { but not }{ }^{(2)} \Sigma_{n+2}\end{array}$ & $\begin{array}{l}\Pi_{n+1} \\
\text { but not }{ }^{(1)} \Sigma_{n+1}\end{array}$ \\
\hline
\end{tabular}

(1) $n=0$, for $\mathbf{B} \Sigma_{1}, \mathbf{I} \Delta_{1}, \mathbf{L} \Delta_{1}^{-}$and $\mathbf{I} \Delta_{1}^{-}$, extensions consistent with $\exp$,

(2) $n=0$, for $\mathbf{B} \Sigma_{1}^{-}$and $\mathbf{U} \mathbf{I} \Delta_{1}$, sound extensions.

- (Descriptive complexity):

\begin{tabular}{|c|c|c|c|c|}
\hline \multirow{2}{*}{ Theories } & $\mathbf{I} \Sigma_{n+1}^{-}$ & $\mathbf{B} \Sigma_{n+1}^{-}+\exp$ & $\begin{array}{c}\mathbf{I} \Pi_{n+1}^{-} \\
\mathbf{L} \Delta_{n+1}^{-}+\exp \\
\mathbf{I} \Delta_{n+1}^{-}+\exp \end{array}$ & $\begin{array}{c}\mathbf{L} \Delta_{1}^{-} \\
\mathbf{I} \Delta_{1}^{-}\end{array}$ \\
\hline type & $n+2 \rightarrow n+2$ & $n+2 \stackrel{w}{\rightarrow} n+2$ & $n+2 \rightarrow n+1$ & $1 \stackrel{w}{\rightarrow} 1$ \\
\hline
\end{tabular}

Previous theorem shows that parameter free fragments do have extensions of quantifier complexity less than that of their axiomatizations (but, necessarily, of big descriptive complexity), while fragments with parameters do not. This divergent behaviour between schemes with and without parameters can be explained from the study of fragments for $\Delta_{n+1}(\mathcal{N})$-formulas (that is, the class of the $\Sigma_{n+1}$-formulas equivalent in the standard model to some $\Pi_{n+1}$-formula). For instance, induction for $\Delta_{n+1}(\mathcal{N})$-formulas, $\mathbf{I} \Delta_{n+1}(\mathcal{N})$, and its parameter free version, $\mathbf{I} \Delta_{n+1}(\mathcal{N})^{-}$, have extensions of less syntactical complexity but of big descriptive complexity. But fragments $\mathbf{I} \Sigma_{n+1}^{-}$and $\mathbf{I} \Delta_{n+1}(\mathcal{N})^{-}$are equivalent, while $\mathbf{I} \Sigma_{n+1}$ is strictly stronger than $\mathbf{I} \Delta_{n+1}(\mathcal{N})$ (for more on $\Delta_{n+1}(\mathcal{N})$-schemes, see [7]).

Notice that, for $n \geq 1$, all the results on the syntactical complexity of extensions are best possible. For $n=0$, it remains to find out if the additional conditions 
(1) and (2) can be omitted. As will be noticed in section 4 (see Corollary 4.5 and remarks following Questions 3 and 4), this question is related to an open problem raised by Wilkie and Paris [21] asking if every model of bounded induction which is not closed under exponentiation is a model of the $\Sigma_{1}$-collection scheme. Results on descriptive complexity are also best possible for $n \geq 1$ (except for the collection scheme). Optimal results for $n=0$ would also yield a solution to Wilkie-Paris' Problem.

Finally, in section 5, from the results in Theorem 1.1, we obtain the following properties on the schemes of induction and minimization for $\Delta_{n+1}$-formulas.

\section{Theorem 1.2.}

(1) $\mathbf{I} \Delta_{n+1}^{-}$and $\mathbf{L} \Delta_{n+1}^{-}$are $\Sigma_{n+2}$-axiomatized, but not $\Pi_{n+2}$.

(2) $\mathbf{U I} \Delta_{n+1}$ is $\bigvee_{n+2}$-axiomatized, but not $\cup_{n+2}$.

(3) $\mathbf{I} \Delta_{n+1}^{-}, \mathbf{L} \Delta_{n+1}^{-}$and $\mathbf{U I} \Delta_{n+1}$ are not finitely axiomatized.

(4) $\mathbf{I} \Delta_{n+1}$ is strictly stronger than $\mathbf{U I} \Delta_{n+1}$ and $\mathbf{U I} \Delta_{n+1}$ is strictly stronger than $\mathbf{I} \Delta_{n+1}^{-}$.

(5) There is no recursively enumerable set of true $\Pi_{n+2}$-sentences which implies $\mathbf{I} \Delta_{n+1}^{-}$.

Some parts of Theorem 1.2 were previously established by Beklemishev [4] by means of proof-theoretical methods. Nevertheless, part 5 above answers problem 4 posed in [4].

Through this paper we also raise some questions which allow one to obtain stronger forms of some of the proved results and we point out relationships between these questions and other open problems in the field of Fragments of Arithmetic.

\section{Preliminaries}

In this section we state some notation and results on fragments of Arithmetics that will be used through this paper (for general notation and references see [12, 14]).

We work in the usual language of first order Arithmetic, $\mathcal{L}=\{0,1,+, \cdot,<\}$. We denote by $\mathcal{N}$ the standard model for $\mathcal{L}$, that is, the model with domain consisting of the set of natural numbers, $\omega$, where the nonlogical symbols have the usual interpretation. For $\mathbf{T}, \mathbf{T}^{\prime}$ theories, we shall write: $\mathbf{T} \Longrightarrow \mathbf{T}^{\prime}$, if $\mathbf{T}$ is an extension of $\mathbf{T}^{\prime} ; \mathbf{T} \Longleftrightarrow \mathbf{T}^{\prime}$, if $\mathbf{T}$ and $\mathbf{T}^{\prime}$ are deductively equivalent; and $\mathbf{T} \Longleftrightarrow \mathbf{T}^{\prime}$, if $\mathbf{T}$ is a proper extension of $\mathbf{T}^{\prime}$. If $\mathcal{N} \models \mathbf{T}$, we say that $\mathbf{T}$ is a sound theory. We denote by $\left\langle x_{1}, x_{2}\right\rangle=y$ Cantor's pairing function and by $(y)_{0}=x$ and $(y)_{1}=x$ its lateral inverse functions; that is, $\left\langle(y)_{0},(y)_{1}\right\rangle=y$. By $x^{y}=z$ we denote a bounded formula which defines, in the standard model, the exponential function; and exp is the $\Pi_{2}$ sentence $\forall x \forall y \exists z\left(x^{y}=z\right)$, see [12] for details.

We recall the usual formulations of the fragments considered in this paper. The induction and minimization axioms for a formula $\varphi(x, \vec{v})$ of $\mathcal{L}$ are, respectively,

$$
\begin{aligned}
& \mathbf{I}_{\varphi}(v) \equiv \varphi(0, \vec{v}) \wedge \forall x[\varphi(x, \vec{v}) \rightarrow \varphi(x+1, \vec{v})] \rightarrow \forall x \varphi(x, \vec{v}) \\
& \mathbf{L}_{\varphi}(v) \equiv \exists x \varphi(x, \vec{v}) \rightarrow \exists x(\varphi(x, \vec{v}) \wedge \forall y<x \neg \varphi(y, \vec{v})) .
\end{aligned}
$$


The collection axiom for a formula $\varphi(x, y, \vec{v})$ of $\mathcal{L}$ is

$$
\mathbf{B}_{\varphi}(z, v) \equiv \forall x \leq z \exists y \varphi(x, y, \vec{v}) \rightarrow \exists u \forall x \leq z \exists y \leq u \varphi(x, y, \vec{v}) .
$$

Let $\Gamma$ be a class of formulas of $\mathcal{L}$. The fragments of induction, minimization and collection for $\Gamma$-formulas are, respectively, the following theories: $\mathbf{I} \Gamma=\mathbf{P}^{-}+\left\{\mathbf{I}_{\varphi}\right.$ : $\varphi \in \Gamma\}, \mathbf{L} \Gamma=\mathbf{P}^{-}+\left\{\mathbf{L}_{\varphi}: \varphi \in \Gamma\right\}$ and $\mathbf{B} \Gamma=\mathbf{I} \Delta_{0}+\left\{\mathbf{B}_{\varphi}: \varphi \in \Gamma\right\}$; where $\mathbf{P}^{-}$ denotes a finite set of $\Pi_{1}$ axioms for the nonnegative part of a commutative discretely ordered ring and $\Delta_{0}$ is the class of the bounded formulas of $\mathcal{L}$.

Now we consider parameter free fragments. We shall write $\varphi\left(x_{1}, \ldots, x_{n}\right) \in \Gamma^{-}$if $\varphi \in \Gamma$ and $x_{1}, \ldots, x_{n}$ are all variables which occur free in $\varphi$. Then $\mathbf{I} \Gamma^{-}$and $\mathbf{L} \Gamma^{-}$are defined as before but restricting the corresponding scheme to formulas $\varphi(x) \in \Gamma^{-}$. We consider two versions of the parameter free collection scheme: $\mathbf{B}_{\mathbf{S}} \Gamma^{-}$is the fragment given by $\mathbf{I} \Delta_{0}+\left\{\mathbf{B}_{\varphi}: \varphi(x, y) \in \Gamma^{-}\right\}$and $\mathbf{B} \Gamma^{-}$is $\mathbf{I} \Delta_{0}$ together with the scheme

$$
\forall x \exists y \varphi(x, y) \rightarrow \forall z \exists u \forall x \leq z \exists y \leq u \varphi(x, y),
$$

for all $\varphi(x, y) \in \Gamma^{-}$. Fragment $\mathbf{B}_{\mathbf{s}} \Gamma^{-}$was first considered in the proof of Proposition 1.7 in [15]. There it is proved that $\mathbf{I} \Sigma_{n+1}^{-} \models \mathbf{B}_{\mathbf{s}} \Sigma_{n+1}^{-} \Longrightarrow \mathbf{B} \Sigma_{n+1}^{-}$and it is posed as an open problem if both formulations of the parameter free collection scheme are equivalent.

Problem 1. $\mathbf{B} \Sigma_{n+1}^{-} \Longrightarrow \mathbf{B}_{\mathbf{s}} \Sigma_{n+1}^{-}$?

We need to consider this (apparently) strong formulation mainly due to the following result.

Lemma 2.1. Let $\varphi(x) \in \Sigma_{n+1}^{-}$. Then $\forall x \leq z \varphi(x) \in \Sigma_{n+1}^{-}$in $\mathbf{B}_{\mathbf{s}} \Sigma_{n+1}^{-}$.

Fragments for $\Delta_{n+1}$-formulas are obtained as follows. The theory $\mathbf{I} \Delta_{n+1}$ is $\mathbf{P}^{-}$ together with

$$
\left\{\forall x(\varphi(x, v) \leftrightarrow \theta(x, v)) \rightarrow \mathbf{I}_{\varphi}(v): \varphi \in \Sigma_{n+1}, \theta \in \Pi_{n+1}\right\} .
$$

If parameters in formulas $\varphi(x)$ and $\theta(x)$ are not allowed, then we obtain $\mathbf{I} \Delta_{n+1}^{-}$. Moreover, Kaye [13] introduced an intermediate case: parameters are allowed but they are uniformly distributed. Namely, $\mathbf{U I} \Delta_{n+1}$ is $\mathbf{P}^{-}$together with

$$
\left\{\forall \vec{v} \forall x(\varphi(x, \vec{v}) \leftrightarrow \theta(x, \vec{v})) \rightarrow \forall \vec{v} \mathbf{I}_{\varphi}(v): \varphi \in \Sigma_{n+1}, \theta \in \Pi_{n+1}\right\} .
$$

Similarly, theories $\mathbf{L} \Delta_{n+1}, \mathbf{L} \Delta_{n+1}^{-}$and $\mathbf{U L} \Delta_{n+1}$ are defined.

For the basic results on the considered fragments we refer the reader to $[12,13,15]$. Nevertheless, we emphasize some properties on $\Delta_{n+1}$-schemes. The relationships between $\Delta_{n+1}$-induction and $\Delta_{n+1}$-minimization schemes are not completely determined. In a preprint (about 1985) H. Friedman claimed $\mathbf{L} \Delta_{n+1}$ and $\mathbf{I} \Delta_{n+1}$ to be equivalent; but in [6] that equivalence appears as an open problem credited to J. Paris. 
- (Paris-Friedman's Conjecture) $\mathbf{I} \Delta_{n+1} \Longleftrightarrow \mathbf{L} \Delta_{n+1}$.

The usual argument for the equivalence of $\mathbf{I} \Sigma_{n+1}$ and $\mathbf{L} \Pi_{n+1}$ allows one to show that the $\Delta_{n+1}$-minimization scheme implies the $\Delta_{n+1}$-induction scheme (for each one of the considered versions), but this argument does not work to prove the converse. Even so, recently T. Slaman [18] has obtained a partial answer to this problem.

Theorem 2.2. (Slaman's Theorem) $\mathbf{I} \Delta_{n+1}+\exp \Longleftrightarrow \mathbf{B} \Sigma_{n+1}+\exp$.

It is well known that $\mathbf{L} \Delta_{n+1} \Longleftrightarrow \mathbf{B} \Sigma_{n+1}$ (it holds that $\mathbf{U L} \Delta_{n+1} \Longleftrightarrow \mathbf{B} \Sigma_{n+1}^{-}$as well) and, for $n \geq 1, \mathbf{I} \Delta_{n+1} \Longrightarrow \mathbf{I} \Sigma_{n} \Longrightarrow \mathbf{e x p}$; so, by Slaman's Theorem, it follows that, for $n \geq 1, \mathbf{I} \Delta_{n+1}$ and $\mathbf{L} \Delta_{n+1}$ are indeed equivalent. However, the case $n=0$ is still an open problem.

Problem 2. $\mathbf{I} \Delta_{1} \Longrightarrow \mathbf{L} \Delta_{1}$ ?

This seems to be a hard question since the proof of Slaman's Theorem heavily depends on the use of the exponential function to handle a (suitable) coding of sequences. Besides it is related to the following open question raised by Wilkie and Paris [21].

Problem 3. $\mathbf{I} \Delta_{0}+\neg \exp \Longrightarrow \mathbf{B} \Sigma_{1}$ ?

Problem 3 in turn is related to a central open question in the field of Fragments of Arithmetic, the End Extension Problem asking if every countable model of $\mathbf{B} \Sigma_{1}$ has a proper end extension to a model of $\mathbf{I} \Delta_{0}$. In [21] it is shown that one of Problem 3 or the End Extension Problem must fail. We also have the following result.

Corollary 2.3. Suppose that Problem 3 has an affirmative answer, that is, $\mathbf{I} \Delta_{0}+$ $\neg \exp \Longrightarrow \mathbf{B} \Sigma_{1}$. Then:

(1) $\mathbf{I} \Delta_{1} \Longleftrightarrow \mathbf{L} \Delta_{1}$.

(2) $\mathbf{I} \Delta_{0}$ is finitely axiomatized if and only if $\mathbf{B} \Sigma_{1}$ is finitely axiomatized.

Proof. (1): By Slaman's Theorem and the hypothesis, it follows that both $\mathbf{I} \Delta_{1}+\mathbf{e x p}$ and $\mathbf{I} \Delta_{1}+\neg \exp$ are extensions of $\mathbf{L} \Delta_{1}$. So, $\mathbf{I} \Delta_{1} \Longrightarrow \mathbf{L} \Delta_{1}$.

(2): Assume that there is a sentence $\varphi$ axiomatizing $\mathbf{I} \Delta_{0}$. Let $\theta$ be a sentence axiomatizing $\mathbf{B} \Sigma_{1}+\mathbf{e x p}$. Then, it holds that $\mathbf{B} \Sigma_{1} \Longleftrightarrow(\varphi \wedge \neg \exp ) \vee \theta$; so $\mathbf{B} \Sigma_{1}$ is finitely axiomatized. The proof of the converse is similar.

Slaman's proof also depends on the presence of parameters in the $\Delta_{n+1}$-schemes; so, it does not provide a direct answer to uniform and parameter free versions of ParisFriedman's Conjecture. Nonetheless, from Slaman's Theorem and a theorem due to Beklemishev [4] stating that $\mathbf{I} \Delta_{1}+\exp$ is $\Sigma_{3}$-conservative over $\mathbf{U I} \Delta_{1}+\exp$, we can deduce a partial answer to the Uniform Conjecture for $n=0$. In [4] the author claims to be routine to prove the analog of the previous conservativeness result for 
schemes $\mathbf{I} \Delta_{n+1}$, UI $\Delta_{n+1}$ for $n \geq 1$. In [9] we have obtained an independent modeltheoretic proof of that fact: for all $n \in \omega, \mathbf{I} \Delta_{n+1}$ is a $\Sigma_{n+3^{-}}$conservative extension of $\mathbf{U I} \Delta_{n+1}$. So, it holds

\section{Theorem 2.4. UI $\Delta_{n+1}+\exp \Longleftrightarrow \mathrm{UL} \Delta_{n+1}+\exp$.}

Therefore, it only remains to answer parameter free Paris-Friedman's Conjecture and its uniform version for $n=0$ :

\section{Problem 4.}

(1) $\mathbf{I} \Delta_{n+1}^{-} \Longrightarrow \mathbf{L} \Delta_{n+1}^{-}$?

(2) $\mathbf{U I} \Delta_{1}^{-} \Longrightarrow \mathbf{U L} \Delta_{1}^{-}$?

Obviously, Theorem 2.4 reduces, for $n \geq 1$, the study of the fragment $\mathbf{U I} \Delta_{n+1}$ to $\mathbf{B} \Sigma_{n+1}^{-}$. Nevertheless, in this paper we shall provide altenative proofs of some properties of $\mathbf{U I} \Delta_{n+1}$ that do not use Theorem 2.4, and also work for $n=0$ without using exponential, see Lemma 3.4, Proposition 4.18 and Theorem 5.3.

Finally, we recall some results on structures constructed using definable elements. Let $\mathfrak{A}$ be a model and $p \in \mathfrak{A}$. Then $\mathcal{K}_{n}(\mathfrak{A}, p)$ is the submodel of $\mathfrak{A}$ with domain $\{a \in$ $\mathfrak{A}: a$ is $\Sigma_{n}$-definable in $\left.(\mathfrak{A}, p)\right\}$ and $\mathcal{I}_{n}(\mathfrak{A}, p)$ is the initial segment of $\mathfrak{A}$ determined by $\mathcal{K}_{n}(\mathfrak{A}, p)$, that is, $\left\{b \in \mathfrak{A}:\right.$ there is $a \in \mathcal{K}_{n}(\mathfrak{A}, p)$ such that $\left.b \leq a\right\}$. If parameter $p$ is not used, we shall write $\mathcal{K}_{n}(\mathfrak{A})$ and $\mathcal{I}_{n}(\mathfrak{A})$, respectively.

Proposition 2.5. ([15, 17]) Let $\mathfrak{A} \models \mathbf{I} \Sigma_{n}$ and $p \in \mathfrak{A}$.

(1) $\mathcal{K}_{n+1}(\mathfrak{A}, p) \prec_{n+1} \mathfrak{A}$ and $\mathcal{K}_{n+1}(\mathfrak{A}, p) \models \mathbf{I} \Sigma_{n}$. Furthermore, if parameter $p$ is not used, $\mathfrak{A}=\mathbf{I} \Sigma_{n}^{-}$suffices.

(2) $\mathcal{K}_{n+1}(\mathfrak{A}, p) \prec_{n+1} \mathcal{I}_{n+1}(\mathfrak{A}, p) \prec_{n} \mathfrak{A}$.

(3) If $\mathcal{I}_{n+1}(\mathfrak{A}, p) \neq \mathfrak{A}$, then $\mathcal{I}_{n+1}(\mathfrak{A}, p) \models \mathbf{B} \Sigma_{n+1}$.

(4) If $\mathfrak{A}=\mathbf{B} \Sigma_{n+1}$, then $\mathcal{I}_{n+1}(\mathfrak{A}, p) \models \mathbf{T h}_{\Pi_{n+2}}(\mathfrak{A})$.

It is well known that submodels constructed using $\Sigma_{n+1}$-definable elements provide examples of models in which the $\Sigma_{n+1}$-induction or the $\Sigma_{n+1}$-collection scheme fails.

Theorem 2.6. (Paris-Kirby, [17]) Let $\mathfrak{A} \models \mathbf{I} \Sigma_{n+1}$ and $p \in \mathfrak{A}$ nonstandard. Then $\mathcal{K}_{n+1}(\mathfrak{A}, p) \not \forall \mathbf{B} \Sigma_{n+1}$ and $\mathcal{I}_{n+1}(\mathfrak{A}, p) \not \models \mathbf{I} \Sigma_{n+1}$.

In [15] it is proved that if parameter $p$ is not used, then $\mathcal{K}_{n+1}(\mathfrak{A}) \models \mathbf{B} \Sigma_{n+1}$ if and only if $\mathcal{K}_{n+1}(\mathfrak{A}) \models \mathbf{B} \Sigma_{n+1}^{-}$, and $\mathcal{I}_{n+1}(\mathfrak{A}) \models \mathbf{I} \Sigma_{n+1}$ if and only if $\mathcal{I}_{n+1}(\mathfrak{A}) \models \mathbf{I} \Pi_{n+1}^{-}$; thus Theorem 2.6 is strengthened as follows.

Theorem 2.7. ([15]) Let $\mathfrak{A} \models \mathbf{I} \Sigma_{n+1}$ such that $\mathcal{K}_{n+1}(\mathfrak{A})$ is nonstandard. Then $\mathcal{K}_{n+1}(\mathfrak{A}) \not \models \mathbf{B} \Sigma_{n+1}^{-}$and $\mathcal{I}_{n+1}(\mathfrak{A}) \not \models \mathbf{I}_{n+1}^{-}$. 
Notice that previous theorem is no longer true when an arbitrary parameter $p$ is allowed. To see this, let us consider $\mathfrak{A}=\mathbf{I} \Sigma_{n+1}+\mathbf{T h}_{\Pi_{n+2}}(\mathcal{N})$ and $p \in \mathfrak{A}$ nonstandard. Then $\mathcal{K}_{n+1}(\mathfrak{A}, p)$ and $\mathcal{I}_{n+1}(\mathfrak{A}, p)$ are models of $\mathbf{T h}_{\Pi_{n+2}}(\mathcal{N})$; and, consequently, both structures satisfy $\mathbf{I} \Sigma_{n+1}^{-}$.

\section{3. $\Sigma_{n+1}$-definable elements}

The main tool for the study of fragments of Arithmetic developed in this paper is the construction of submodels using definable elements. It will be important to establish the usual properties of these structures under sufficiently general conditions. Concretely, the aim of this section is to refine Theorems 2.6 and 2.7 in two ways:

(a) weakening the main hypothesis on $\mathfrak{A}, \mathfrak{A} \models \mathbf{I} \Sigma_{n+1}$; and

(b) characterizing parameters $p \in \mathfrak{A}$ such that $\mathcal{K}_{n+1}(\mathfrak{A}, p) \not \models \mathbf{B} \Sigma_{n+1}^{-}$.

To this end, following ideas in $[10,15]$, we consider classes of $\Pi_{n+1}$-definable and $\Pi_{n+1}-$ minimal elements.

(-) We say that $a \in \mathfrak{A}$ is $\Pi_{k}$-definable in $(\mathfrak{A}, p)$ if there exists $\varphi(x, v) \in \Pi_{k}$ such that

$$
\mathfrak{A}=\varphi(a, p) \wedge \forall x(\varphi(x, p) \rightarrow x=a) .
$$

$(-)$ We say that $a \in \mathfrak{A}$ is $\Pi_{k}-$ minimal in $(\mathfrak{A}, p)$ if there exists $\varphi(x, v) \in \Pi_{k}$ such that

$$
\mathfrak{A} \models \varphi(a, p) \wedge \forall x<a \neg \varphi(x, p),
$$

that is, $\mathfrak{A}=a=(\mu x)(\varphi(x, p))$.

We shall denote by $\mathcal{D}_{k}(\mathfrak{A}, p)$ and $\mathcal{M}_{k}(\mathfrak{A}, p)$, respectively, the classes of $\Pi_{k}$-definable and $\Pi_{k}$-minimal elements in $(\mathfrak{A}, p)$. If no parameters are used, we shall write $\mathcal{D}_{k}(\mathfrak{A})$ and $\mathcal{M}_{k}(\mathfrak{A})$. If $\mathfrak{A}=\mathbf{B} \Sigma_{k+1}$ then $\mathcal{D}_{k}(\mathfrak{A}, p)$ and $\mathcal{M}_{k}(\mathfrak{A}, p)$ are domains of substructures of $\mathfrak{A}$, but they are not, in general, models of very weak fragments of Arithmetic. In fact, if they are nonstandard, then they are not closed under Cantor's lateral inverse functions; so, they are not models of Open induction.

Our starting point is the study of the distribution of definable elements. We write $\mathcal{A} \subseteq{ }^{e} \mathcal{B}$ if $\mathcal{A}$ is an initial segment of $\mathcal{B}$, and $\mathcal{A} \subseteq \subseteq^{c} \mathcal{B}$ if $\mathcal{A}$ is a cofinal subclass of $\mathcal{B}$.

Proposition 3.1. Let $\mathfrak{A} \models \mathbf{I} \Sigma_{n+1}$ and $p \in \mathfrak{A}$. Then

$$
\mathcal{K}_{n+1}(\mathfrak{A}, p)^{e} \mathcal{D}_{n+1}(\mathfrak{A}, p) \subseteq{ }^{c} \mathcal{M}_{n+1}(\mathfrak{A}, p) \subseteq \subseteq^{c} \mathcal{K}_{n+2}(\mathfrak{A}, p) .
$$

Furthermore, if parameter $p$ is not used, $\mathfrak{A} \models \mathbf{I} \Sigma_{n+1}^{-}$suffices.

Proof. $\left(\mathcal{K}_{n+1}(\mathfrak{A}, p) \subseteq e \mathcal{D}_{n+1}(\mathfrak{A}, p)\right)$ : Let $a \in \mathcal{K}_{n+1}(\mathfrak{A}, p)$ and $\varphi(x, v) \in \Sigma_{n+1}$ a formula defining $a$ in $(\mathfrak{A}, p)$. Then $\forall z(\varphi(z, v) \rightarrow x=z)$ is a $\Pi_{n+1}$ formula that defines $a$ in $(\mathfrak{A}, p)$; so, $a \in \mathcal{D}_{n+1}(\mathfrak{A}, p)$. Let $b \in \mathcal{D}_{n+1}(\mathfrak{A}, p)$ such that $b \leq a$ and $\psi(x, v) \in \Pi_{n+1}$ a formula defining $b$ in $(\mathfrak{A}, p)$. Let $\delta(x, v)$ be the formula

$$
\exists z(\varphi(z, v) \wedge \forall y \leq z(\psi(y, v) \rightarrow y=x)) .
$$


Then $\delta(x, v) \in \Sigma_{n+1}$ in $\mathbf{B} \Sigma_{n+1}$ and, as $b \leq a, \mathfrak{A} \models \delta(b, p) \wedge \forall x(\delta(x, p) \rightarrow x=b)$. So, $b \in \mathcal{K}_{n+1}(\mathfrak{A}, p)$.

$\left(\mathcal{D}_{n+1}(\mathfrak{A}, p) \subseteq{ }^{c} \mathcal{M}_{n+1}(\mathfrak{A}, p)\right)$ : The proof of this part essentially appears in the proof of Proposition 1.13 in [15]. Let $a \in \mathcal{D}_{n+1}(\mathfrak{A}, p)$ and $\varphi(x, v) \in \Pi_{n+1}$ a formula defining $a$ in $(\mathfrak{A}, p)$. Then $\mathfrak{A}=a=(\mu x)(\varphi(x, p))$; so, $a \in \mathcal{M}_{n+1}(\mathfrak{A}, p)$. Now let $b \in \mathcal{M}_{n+1}(\mathfrak{A}, p)$ and $\psi(x, y, v) \in \Sigma_{n}$ such that $\mathfrak{A} \models b=(\mu x)(\forall y \psi(x, y, p))$. Since $\mathfrak{A} \models \mathbf{B} \Sigma_{n+1}$,

$$
\mathfrak{A} \models \forall y \psi(b, y, p) \wedge \exists u \forall x<b \exists y \leq u \neg \psi(x, y, p) .
$$

So, as $\mathfrak{A}=\mathbf{L} \Pi_{n}\left(\Longleftrightarrow \mathbf{I} \Sigma_{n}\right)$, there exists $c \in \mathfrak{A}$ such that

$$
\mathfrak{A}=c=(\mu u)(\forall x<b \exists y \leq u \neg \psi(x, y, p)) ;
$$

that is, $c$ is the maximum of the function $\neg \psi(x, y, p)$ when $x<b$. Let $d=\langle b, c\rangle$ and let $\theta(u, v)$ be the formula

$$
\forall y \psi\left((u)_{0}, y, v\right) \wedge \forall x<(u)_{0} \exists y \leq(u)_{1} \neg \psi(x, y, v) \wedge \exists x<(u)_{0} \forall y<(u)_{1} \psi(x, y, v) .
$$

Since $\theta(u, v) \in \Pi_{n+1}$ in $\mathbf{B} \Sigma_{n+1}$ and $\mathfrak{A} \models \theta(d, p) \wedge \forall u(\theta(u, p) \rightarrow u=d), d \in$ $\mathcal{D}_{n+1}(\mathfrak{A}, p)$. Hence, as $b=(d)_{0} \leq d$, this proves that $\mathcal{D}_{n+1}(\mathfrak{A}, p)$ is cofinal in $\mathcal{M}_{n+1}(\mathfrak{A}, p)$.

$\left(\mathcal{M}_{n+1}(\mathfrak{A}, p) \subseteq \subseteq^{c} \mathcal{K}_{n+2}(\mathfrak{A}, p)\right)$ : Let $a \in \mathcal{M}_{n+1}(\mathfrak{A}, p), \varphi(x, v) \in \Pi_{n+1}$ such that $\mathfrak{A} \models$ $a=(\mu x)(\varphi(x, p))$ and $\theta(x, v) \equiv \varphi(x, v) \wedge \forall z<x \neg \varphi(z, v)$. Then $\theta(x, v) \in \Sigma_{n+2}$ in $\mathbf{B} \Sigma_{n+1}$ and $\mathfrak{A}=\theta(a, p) \wedge \forall x(\theta(x, p) \rightarrow x=a)$. So, $a \in \mathcal{K}_{n+2}(\mathfrak{A}, p)$. Now let $a \in \mathcal{K}_{n+2}(\mathfrak{A}, p)$ and $\varphi(x, y, v) \in \Pi_{n+1}$ such that $\exists y \varphi(x, y, v)$ defines $a$ in $(\mathfrak{A}, p)$. So, $\mathfrak{A}=\exists u \varphi\left((u)_{0},(u)_{1}, p\right)$. Since $\mathfrak{A} \models \mathbf{L} \Pi_{n+1}$, there exists $b \in \mathfrak{A}$ such that

$$
\mathfrak{A}=b=(\mu u)\left(\varphi\left((u)_{0},(u)_{1}, p\right)\right) .
$$

So, $b \in \mathcal{M}_{n+1}(\mathfrak{A}, p)$. Since $a=(b)_{0}<b, \mathcal{M}_{n+1}(\mathfrak{A}, p)$ is cofinal in $\mathcal{K}_{n+2}(\mathfrak{A}, p)$.

Nota 3.2 .

(1) Notice that the hypothesis $\mathfrak{A} \models \mathbf{I} \Sigma_{n+1}$ is only needed to establish that $\mathcal{M}_{n+1}(\mathfrak{A}, p)$ is cofinal in $\mathcal{K}_{n+2}(\mathfrak{A}, p)$. For the rest of the proof of Proposition $3.1, \mathfrak{A}=\mathbf{B} \Sigma_{n+1}$ (or, $\mathfrak{A}=\mathbf{B}_{\mathbf{s}} \Sigma_{n+1}^{-}$if parameter $p$ is not used) suffices.

(2) The proof of the last inclusion in the previous proposition also shows that if $\mathfrak{A} \models \mathbf{I} \Delta_{0}$ and $p \in \mathfrak{A}$, then $\mathcal{M}_{0}(\mathfrak{A}, p)$ is cofinal in $\mathcal{K}_{1}(\mathfrak{A}, p)$.

(3) It is well known that $\Sigma_{n+1}$-definable elements are not cofinal in nonstandard models of $\mathbf{I} \Sigma_{n+1}$. Hence, by Proposition 3.1, it follows that in every model of $\mathbf{I} \Sigma_{n+1}^{-}$containing nonstandard $\Sigma_{n+2}$-definable elements there exists a $\Pi_{n+1^{-}}$ definable element which is not $\Sigma_{n+1}$-definable. Even more; it is easy to check that for every model of $\mathbf{I} \Sigma_{n+1}$ containing nonstandard $\Sigma_{n+1}$-definable elements all the inclusions in Proposition 3.1 are proper. 
As first observed by $\mathrm{H}$. Lessan, in $\mathfrak{A} \models \mathbf{I} \Sigma_{n}^{-}$, there exist nonstandard $\Sigma_{n+1^{-}}$ definable elements if and only if $\mathfrak{A} \not \models \mathbf{T h}_{\Pi_{n+1}}(\mathcal{N})$. So, from Proposition 3.1 we obtain the following corollary.

Corollary 3.3. Let $\mathfrak{A}=\mathbf{I} \Sigma_{n+1}^{-}$. The following conditions are equivalent.

(1) $\mathfrak{A} \not \models \mathbf{T h}_{\Pi_{n+2}}(\mathcal{N})$.

(2) $\mathcal{K}_{n+2}(\mathfrak{A})$ is nonstandard.

(3) $\mathcal{D}_{n+1}(\mathfrak{A})$ is nonstandard.

(4) $\mathcal{M}_{n+1}(\mathfrak{A})$ is nonstandard.

Consequently, in models of $\mathbf{I} \Sigma_{n+1}^{-}, \mathfrak{A} \not \models \mathbf{T h}_{\Pi_{n+2}}(\mathcal{N})$ is a necessary and sufficient condition for the existence of nonstandard $\Pi_{n+1}$-definable elements. Next question asks if the hypothesis on $\mathfrak{A}$ can be weakened:

Question 1. Let $\mathfrak{A}=\mathbf{B} \Sigma_{n+1}$ such that $\mathfrak{A} \not \mathbf{T h}_{\Pi_{n+2}}(\mathcal{N})$. Is $\mathcal{D}_{n+1}(\mathfrak{A})$ nonstandard?

A positive answer to this question would yield an improvement of our results on the descriptive complexity of extensions of $\mathbf{B} \Sigma_{n+1}^{-}$, see Proposition 4.17.

Next result shows conditions under which models of definable elements satisfying some parameter free scheme also satisfy its parameter counterpart.

\section{Lemma 3.4.}

(1) If $\mathcal{K}_{n+1}(\mathfrak{B})=\mathfrak{B}$ and $\mathfrak{B}=\mathbf{I} \Delta_{n+1}^{-}$, then $\mathfrak{B} \models \mathbf{I} \Delta_{n+1}$. If, moreover, $\mathfrak{B} \models \mathbf{e x p}$, then, by Slaman's Theorem (see Theorem 2.2), $\mathfrak{B} \models \mathbf{B} \Sigma_{n+1}$.

(2) Assume that $\mathcal{K}_{n+2}(\mathfrak{B})=\mathfrak{B}$.

(a) If $\mathfrak{B} \models \mathbf{B} \Sigma_{n+1}^{-}$then $\mathfrak{B} \models \mathbf{B} \Sigma_{n+1}$.

(b) If $\mathfrak{B}=\mathbf{U I} \Delta_{n+1}$ then $\mathfrak{B}=\mathbf{I} \Delta_{n+1}$.

Proof. (1): Let $\varphi(x, v) \in \Sigma_{n+1}, \theta(x, v) \in \Pi_{n+1}$ and $b \in \mathfrak{B}$ such that $\mathfrak{B}=\varphi(x, b) \leftrightarrow$ $\theta(x, b)$ and $\mathfrak{B} \models \varphi(0, b) \wedge(\varphi(x, b) \rightarrow \varphi(x+1, b))$. Since all elements of $\mathfrak{B}$ are $\Sigma_{n+1^{-}}$ definable, there exists $\delta(v) \in \Sigma_{n+1}^{-}$defining $b$ in $\mathfrak{B}$. Let $\varphi^{\prime}(x) \equiv \exists v(\delta(v) \wedge \varphi(x, v))$. Then, $\mathfrak{B}=\exists v(\delta(v) \wedge \varphi(x, v)) \leftrightarrow \forall v(\delta(v) \rightarrow \varphi(x, v))$ and $\mathfrak{B}=\varphi^{\prime}(0) \wedge\left(\varphi^{\prime}(x) \rightarrow\right.$ $\left.\varphi^{\prime}(x+1)\right)$. Since $\mathfrak{B}=\mathbf{I} \Delta_{n+1}^{-}, \mathfrak{B}=\forall x \varphi^{\prime}(x)$. So, $\mathfrak{B} \models \forall x \varphi(x, b)$, as required.

(2-(a)): The proof of this part implicitly appears in the proof of Proposition 1.13 in [15]. Let $\varphi(x, y, v) \in \Pi_{n}^{-}$and $a, b \in \mathfrak{B}$ such that $\mathfrak{B} \models \forall x \exists y \varphi(x, y, b)$. Let $\theta\left(v, z_{1}, z_{2}\right) \in \Sigma_{n}$ such that $\exists z_{1} \forall z_{2} \theta\left(v, z_{1}, z_{2}\right)$ defines $b$ in $\mathfrak{B}$. Since $\mathfrak{B} \models \mathbf{B} \Sigma_{n+1}^{-}$and $\mathfrak{B} \models \forall v, x, z_{1} \exists y, z_{2}\left(\neg \theta\left(v, z_{1}, z_{2}\right) \vee \varphi(x, y, v)\right)$, then it follows that

$$
\mathfrak{B} \models \forall v, z_{1} \exists u \forall x \leq a \exists y, z_{2} \leq u\left(\neg \theta\left(v, z_{1}, z_{2}\right) \vee \varphi(x, y, v)\right) .
$$

So, $\mathfrak{B} \models \exists u \forall x \leq a \exists y \leq u \varphi(x, y, b)$, as required.

$(2-(\mathrm{b}))$ : For $n>0$ the result follows from Theorem 2.4 and part $2-(\mathrm{a})$. Nevertheless we offer an alternative proof which works also for $n=0$. 
Let $\varphi(x, y, v) \in \Pi_{n}, \psi(x, y, v) \in \Sigma_{n}$ and $b \in \mathfrak{B}$ such that $\mathfrak{B} \models \exists y \varphi(x, y, b) \leftrightarrow$ $\forall y \psi(x, y, b)$ and

$$
\mathfrak{B} \models \exists y \varphi(0, y, b) \quad \text { and } \quad \mathfrak{B} \models \exists y \varphi(x, y, b) \rightarrow \exists y \varphi(x+1, y, b) .
$$

Let us see that $\mathfrak{B} \models \forall x \exists y \varphi(x, y, b)$.

Since $\mathcal{K}_{n+2}(\mathfrak{B})=\mathfrak{B}$, there exists $\delta(w, y, v) \in \Sigma_{n}$ such that $\exists w \forall y \delta(w, y, v) \in \Sigma_{n+2}$ defines $b$ in $\mathfrak{B}$. Let $\varphi_{0}(x, y, w, v) \in \Pi_{n}$ be the formula $\neg \delta(w, y, v) \vee \varphi(x, y, v) \vee$ $\neg \psi(x, y, v)$. Let $\varphi_{1}(x, y, w, v) \in \Sigma_{n+1}$ (recall that, for $n>0$, $\mathbf{U I} \Delta_{n+1} \Longrightarrow \mathbf{B} \Sigma_{n}$ ) such that

$$
\mathfrak{B} \models \varphi_{1}(x, y, w, v) \leftrightarrow\left[\varphi_{0}(x, y, w, v) \wedge \forall z<y \neg \varphi_{0}(x, z, w, v)\right] .
$$

Let $\varphi_{2}(x, w, v) \in \Sigma_{n+1}$ be the formula $\exists y\left[\varphi_{1}(x, y, w, v) \wedge \varphi(x, y, v)\right]$. Since $\mathfrak{B} \models$ $\forall x, w, v \exists y \varphi_{0}(x, y, w, v)$ and $\mathfrak{B} \models \mathbf{I} \Sigma_{n}, \mathfrak{B} \models \exists ! y \varphi_{1}(x, y, w, v) ;$ so, $\mathfrak{B} \models \varphi_{2}(x, w, v) \leftrightarrow$ $\forall y\left[\varphi_{1}(x, y, w, v) \rightarrow \varphi(x, y, v)\right]$. Hence, $\varphi_{2}(x, w, v) \in \Delta_{n+1}(\mathfrak{B})$. Let $c \in \mathfrak{B}$ such that $\mathfrak{B} \models \forall y \delta(c, y, b)$. Then $\mathfrak{B}=\varphi_{2}(x, c, b) \leftrightarrow \exists y \varphi(x, y, b)$. So,

$$
\mathfrak{B}=\varphi_{2}(0, c, b) \quad \text { and } \quad \mathfrak{B}=\varphi_{2}(x, c, b) \rightarrow \varphi_{2}(x+1, c, b) .
$$

Since $\mathfrak{B} \models \mathbf{U I} \Delta_{n+1}, \mathfrak{B} \models \forall x \varphi_{2}(x, c, b)$ and, as a consequence, $\mathfrak{B} \models \forall x \exists y \varphi(x, y, b)$, as required.

We can now obtain the main result of this section: the desired generalization of Theorems 2.6 and 2.7 .

\section{Theorem 3.5.}

(1) Let $\mathfrak{A}=\mathbf{I} \Sigma_{n}$ and $p \in \mathfrak{A}$ nonstandard. Then $\mathcal{K}_{n+1}(\mathfrak{A}, p) \not \models \mathbf{B} \Sigma_{n+1}+\mathbf{e x p}$.

(2) If $\mathfrak{A}=\mathbf{I} \Sigma_{n}^{-}$and $\mathcal{K}_{n+1}(\mathfrak{A})$ is nonstandard then $\mathcal{K}_{n+1}(\mathfrak{A}) \not \models \mathbf{I} \Delta_{n+1}^{-}+$exp.

(3) Let $\mathfrak{A} \models \mathbf{B}_{\mathbf{s}} \Sigma_{n+1}^{-}$and $p \in \mathcal{M}_{n+1}(\mathfrak{A})$ nonstandard. Then $\mathcal{K}_{n+1}(\mathfrak{A}, p) \not \models$ $\mathbf{B} \Sigma_{n+1}^{-}+$exp.

Proof. (1): Towards a contradiction assume that $\mathcal{K}_{n+1}(\mathfrak{A}, p) \models \mathbf{B} \Sigma_{n+1}+\mathbf{e x p}$. Let $a \in \mathcal{K}_{n+1}(\mathfrak{A}, p)$ nonstandard. Let us consider the following cases.

Case A: $n \geq 1$. Let $\mathbf{S a t}_{\Pi_{n}}(x) \in \Pi_{n}$ be a truth definition for $\Pi_{n}$ formulas in $\mathbf{I} \Sigma_{1}$. By $\mathcal{K}_{n+1}(\mathfrak{A}, p) \models \mathbf{B} \Sigma_{n+1}$ and the fact that $\mathcal{M}_{n}(\mathfrak{A}, p)$ is cofinal in $\mathcal{K}_{n+1}(\mathfrak{A}, p)$ (see Proposition 3.1), it follows that there exists $c \in \mathcal{K}_{n+1}(\mathfrak{A}, p)$ such that $\mathcal{K}_{n+1}(\mathfrak{A}, p)$ satisfies

$$
\forall u \leq a \exists \sigma<a \exists x<c\left(\forall z<x \neg \operatorname{Sat}_{\Pi_{n}}(\sigma(z, p)) \wedge \operatorname{Sat}_{\Pi_{n}}(\sigma(x, p)) \wedge u=(x)_{0}\right) .
$$

So, there exists an injective $\Sigma_{0}\left(\Sigma_{n}\right)-$ map from $2^{a+1}-1$ into $2^{a}-1$. This contradicts the Pigeon-hole principle for (coded) $\Sigma_{0}\left(\Sigma_{n}\right)$-functions in models of $\mathbf{I} \Sigma_{n}, n \geq 1$.

Case B: $n=0$. Let $d \in \mathcal{K}_{1}(\mathfrak{A}, p)$ nonstandard. By the proof of Proposition 3.1 (see Remark 3.2-(2)), it holds that $\mathcal{M}_{0}(\mathfrak{A}, p)$ is cofinal in $\mathcal{K}_{1}(\mathfrak{A}, p)$; so, $\mathcal{K}_{1}(\mathfrak{A}, p)$ satisfies

$\forall u \leq a \exists \sigma<a \exists x\left(\forall z<x \neg \mathcal{V}_{0}\left(\sigma(z, p), 2^{(z+p+2)^{d}}\right) \wedge \mathcal{V}_{0}\left(\sigma(x, p), 2^{(z+p+2)^{d}}\right) \wedge u=(x)_{0}\right)$, where $\mathcal{V}_{0}\left(v_{1}, v_{2}\right) \in \Delta_{0}$ is a truth definition for $\Delta_{0}$ formulas in $\mathbf{I} \Delta_{0}+\exp$, see [12]. Since $\mathcal{K}_{1}(\mathfrak{A}, p) \models \mathbf{B} \Sigma_{1}$, there is $c \in \mathcal{K}_{1}(\mathfrak{A}, p)$ such that $\mathcal{K}_{1}(\mathfrak{A}, p)$ satisfies 


$$
\forall u \leq a \exists \sigma<a \exists x, y<c\left\{\begin{array}{l}
y=2^{(x+p+2)^{d}} \wedge \mathcal{V}_{0}(\sigma(x, p), y) \wedge u=(x)_{0} \wedge \\
\forall z<x \exists y_{1} \leq y\left[y_{1}=2^{(z+p+2)^{d}} \wedge \neg \mathcal{V}_{0}\left(\sigma(z, p), y_{1}\right)\right] .
\end{array}\right.
$$

So, there exists an injective $\Delta_{0}-$ map from $2^{a+1}-1$ into $2^{a}-1$. This contradicts the Pigeon-hole principle for (coded) $\Delta_{0}$-functions in models of $\mathbf{I} \Delta_{0}+\mathbf{e x p}$.

(2): Assume that $\mathcal{K}_{n+1}(\mathfrak{A}) \models \mathbf{I} \Delta_{n+1}^{-}+\exp$. Then, by Lemma $3.4-(1)$ and the fact that all elements of $\mathcal{K}_{n+1}(\mathfrak{A})$ are $\Sigma_{n+1}$-definable, it follows that $\mathcal{K}_{n+1}(\mathfrak{A}) \models \mathbf{B} \Sigma_{n+1}+$ exp. Thus, the same reasoning as in part (1) gives the required contradiction. Notice that now $\mathfrak{A}=\mathbf{I} \Sigma_{n}^{-}$suffices since parameter $p$ is not used.

(3): First observe that $\mathbf{B}_{\mathbf{s}} \Sigma_{n+1}^{-} \Longrightarrow \mathbf{I} \Sigma_{n}$; so, $\mathcal{K}_{n+1}(\mathfrak{A}, p) \prec_{n+1} \mathfrak{A}$ and, by part (1), $\mathcal{K}_{n+1}(\mathfrak{A}, p) \not \models \mathbf{B} \Sigma_{n+1}+$ exp. Now we check that $\mathcal{K}_{n+1}(\mathfrak{A}, p)$ verifies the hypothesis of Lemma 3.4-(2).

Claim $\mathcal{K}_{n+2}\left(\mathcal{K}_{n+1}(\mathfrak{A}, p)\right)=\mathcal{K}_{n+1}(\mathfrak{A}, p)$.

Proof of Claim. Let $a \in \mathcal{K}_{n+1}(\mathfrak{A}, p)$ and $\varphi(x, v) \in \Sigma_{n+1}$ a formula defining $a$ in $(\mathfrak{A}, p)$. Since $p \in \mathcal{M}_{n+1}(\mathfrak{A})$, there exists $\theta(v, y) \in \Sigma_{n}$ such that $\mathfrak{A}=p=(\mu v)(\forall y \theta(v, y))$. Since $\mathfrak{A} \models \mathbf{B}_{\mathbf{s}} \Sigma_{n+1}^{-}$,

$$
\mathfrak{A} \models \forall y \theta(p, y) \wedge \exists u \forall v<p \exists y \leq u \neg \theta(v, y) ;
$$

and, therefore, $\mathcal{K}_{n+1}(\mathfrak{A}, p)$ also satisfies this formula. Let $\delta(x)$ be the formula

$$
\exists v(\forall y \theta(v, y) \wedge \exists u \forall w<v \exists y \leq u \neg \theta(w, y) \wedge \varphi(x, v)) .
$$

Then, $\delta(x)$ is $\Sigma_{n+2}^{-}$in $\mathbf{I} \Sigma_{n}$ and defines $a$ in $\mathcal{K}_{n+1}(\mathfrak{A}, p)$. Which proves the claim.

Thus, part (3) follows from previous claim, Lemma 3.4-(2) and the fact that $\mathcal{K}_{n+1}(\mathfrak{A}, p)$ is not a model of $\mathbf{B} \Sigma_{n+1}+\mathbf{e x p}$.

We close this section with a result concerning relationships between models of $\Sigma_{n+1}$-definable elements and the fragment $\mathbf{I} \Pi_{n+1}^{-}$.

Lemma 3.6. Let $\mathbf{T}=\left\{\exists x \theta_{i}(x): \theta_{i}(x) \in \Pi_{n+1}^{-}, i \in \omega\right\}$ be a $\Sigma_{n+2}$-axiomatized theory and $\mathfrak{A}=\mathbf{T}+\mathbf{I} \Sigma_{n+1}^{-}$such that:

(i) $\mathfrak{A} \not \mathbf{T h}_{\Pi_{n+2}}(\mathcal{N})$.

(ii) $\mathbf{T} \in \operatorname{SSy}\left(\mathcal{K}_{n+2}(\mathfrak{A})\right)$ (where $\operatorname{SSy}\left(\mathcal{K}_{n+2}(\mathfrak{A})\right)$ is the standard system of $\mathcal{K}_{n+2}(\mathfrak{A})$ ).

(iii) $\left\{a_{i}: i \in \omega, \mathfrak{A} \models a_{i}=(\mu x)\left(\theta_{i}(x)\right)\right\}$ is not cofinal in $\mathcal{K}_{n+2}(\mathfrak{A})$.

Then there exists $p \in \mathcal{D}_{n+1}(\mathfrak{A})$ such that, for each $\mathfrak{B} \prec_{n} \mathfrak{A}$, if $p \in \mathfrak{B}$, then $\mathfrak{B}=\mathbf{T}$.

Proof. By Corollary 3.3 and (i), $\mathcal{K}_{n+2}(\mathfrak{A})$ is nonstandard; and, since $\mathcal{K}_{n+2}(\mathfrak{A}) \prec_{n+2}$ $\mathfrak{A}$, by (iii), there is $a \in \mathcal{K}_{n+2}(\mathfrak{A})$ such that, for every $i \in \omega, \mathcal{K}_{n+2}(\mathfrak{A}) \models \exists x \leq a \theta_{i}(x)$. By (ii), there exists $b \in \mathcal{K}_{n+2}(\mathfrak{A})$ codifying $\left\{\left\ulcorner\theta_{i}(x)\right\urcorner: i \in \omega\right\}$, where $\left\ulcorner\theta_{i}(x)\right\urcorner$ denotes the Gödel number of $\theta_{i}(x)$. Then, for each $k \in \omega$, we have (notice that $(x)_{y}=z$ denotes a $\Delta_{0}$ formula defining the standard coding of sequences in $\mathbf{I} \Delta_{0}+\exp$ )

$$
\mathcal{K}_{n+2}(\mathfrak{A}) \models \forall \sigma \leq k \exists x \leq a\left[(b)_{\sigma} \neq 0 \rightarrow \operatorname{Sat}_{\Pi_{n+1}}(\sigma(x))\right] .
$$


Let $a^{\prime} \in \mathcal{K}_{n+2}(\mathfrak{A})$ be a sequence number of nonstandard length such that $\mathcal{K}_{n+2}(\mathfrak{A}) \models$ $\forall v<\lg \left(a^{\prime}\right)\left(\left(a^{\prime}\right)_{v}=a\right)$. Then, for each $k \in \omega$,

$$
\mathcal{K}_{n+2}(\mathfrak{A}) \models \exists u \leq a^{\prime} \forall \sigma \leq k\left[(b)_{\sigma} \neq 0 \rightarrow \operatorname{Sat}_{\Pi_{n+1}}\left(\sigma\left((u)_{\sigma}\right)\right)\right] .
$$

Since $\mathcal{K}_{n+2}(\mathfrak{A}) \models \mathbf{I} \Sigma_{n+1}$, by overspill, there exist $c \in \mathcal{K}_{n+2}(\mathfrak{A})$ nonstandard and $d \in$ $\mathcal{K}_{n+2}(\mathfrak{A})$ such that $d \leq a^{\prime}$ and $\mathcal{K}_{n+2}(\mathfrak{A}) \models \forall \sigma \leq c\left[(b)_{\sigma} \neq 0 \rightarrow \operatorname{Sat}_{\Pi_{n+1}}\left(\sigma\left((d)_{\sigma}\right)\right)\right]$. From the proof of Proposition 3.1, it follows that there is $p \in \mathcal{D}_{n+1}(\mathfrak{A})$ such that $(p)_{0,0}=d$.

Now let $\mathfrak{B} \prec_{n} \mathfrak{A}$ such that $p \in \mathfrak{B}$. Let $\exists x \theta_{i}(x)$ be a nonlogical axiom of $\mathbf{T}$ and $k=\left\ulcorner\theta_{i}(x)\right\urcorner$. Then $\mathcal{K}_{n+2}(\mathfrak{A}) \models \operatorname{Sat}_{\Pi_{n+1}}\left(\left\ulcorner\theta_{i}\left((d)_{k}\right)\right\urcorner\right)$; so, as $\mathcal{K}_{n+2}(\mathfrak{A}) \models \mathbf{I} \Sigma_{1}$, $\mathcal{K}_{n+2}(\mathfrak{A}) \models \theta_{i}\left((d)_{k}\right)$. Then, by $\mathcal{K}_{n+2}(\mathfrak{A}) \prec_{n+2} \mathfrak{A}$ and $\theta_{i}(x) \in \Pi_{n+1}^{-}$, we have $\mathfrak{A} \models$ $\theta_{i}\left((d)_{k}\right)$. Now, since $\mathfrak{B} \prec_{n} \mathfrak{A}$ and $(d)_{k}=(p)_{0,0, k} \in \mathfrak{B}, \mathfrak{B} \models \theta_{i}\left((d)_{k}\right)$. So, $\mathfrak{B} \models \mathbf{T}$, as required.

Corollary 3.7. Let $\mathfrak{A}=\mathbf{I} \Sigma_{n+1}^{-}$such that $\mathfrak{A} \not \models \mathbf{T h}_{\Pi_{n+2}}(\mathcal{N})$. There exists $p \in \mathcal{D}_{n+1}(\mathfrak{A})$ nonstandard such that for every $a \in \mathfrak{A}, \mathcal{K}_{n+1}(\mathfrak{A}, a, p) \models \mathbf{I}_{n+1}^{-}$.

Proof. Let $\left\{\varphi_{i}(u) \in \Sigma_{n+1}^{-}: i \in \omega\right\}$ be a recursive enumeration of all the $\Sigma_{n+1}$ formulas in which only the variable $u$ occurs free. For each $i \in \omega$, let $\theta_{i}(x) \in \Pi_{n+1}^{-}$ be a formula such that

$$
\exists x \theta_{i}(x) \equiv \exists x\left(\exists u \varphi_{i}(u) \rightarrow x=(\mu u)\left(\varphi_{i}(u)\right)\right) .
$$

Then $\mathbf{I}_{n+1}^{-} \Longleftrightarrow \mathbf{L} \Sigma_{n+1}^{-} \Longleftrightarrow \mathbf{P}^{-}+\left\{\exists x \theta_{i}(x): i \in \omega\right\}$. As $\left\{\theta_{i}(x): i \in \omega\right\}$ is recursive, it is coded in $\operatorname{SSy}\left(\mathcal{K}_{n+2}(\mathfrak{A})\right)$. Since $\mathfrak{A} \models \mathbf{I}_{n+1}^{-}$and $\mathcal{K}_{n+1}(\mathfrak{A}) \prec_{n+1} \mathfrak{A}$, then for each $i \in \omega$ it holds that there exists $b_{i} \in \mathcal{K}_{n+1}(\mathfrak{A})$ such that $\mathcal{K}_{n+1}(\mathfrak{A}) \models$ $\exists x \leq b_{i} \theta_{i}(x)$. Let $b \in \mathcal{K}_{n+2}(\mathfrak{A})$ such that, for every $c \in \mathcal{K}_{n+1}(\mathfrak{A}), c \leq b$. Then, for each $i \in \omega, \mathcal{K}_{n+2}(\mathfrak{A}) \models \exists x \leq b \theta_{i}(x)$. Let $p \in \mathcal{D}_{n+1}(\mathfrak{A})$ as in the proof of Lemma 3.6. Then $\mathcal{K}_{n+1}(\mathfrak{A}, a, p) \models \mathbf{I}_{n+1}^{-}$.

\section{Extensions of bounded complexity}

In this section we systematically study the syntactical and descriptive complexity of extensions of fragments of Arithmetic. We shall see that fragments with parameters do not have consistent extensions of syntactical complexity less than that of their natural formulation, whereas parameter free fragments do. Then we shall study the descriptive complexity of such extensions of parameter free schemes.

We first establish some elementary properties of extensions of $\mathbf{T h}_{\Pi_{n+1}}(\mathcal{N})$, the theory of the $\Pi_{n+1}-$ sentences true in the standard model. A theory $\mathbf{T}$ is said to be $\Gamma$-definable if there exists a formula $\varphi(x) \in \Gamma$ which defines in the standard model the class of the Gödel numbers of the axioms of $\mathbf{T}$.

Lemma 4.1. Let $\mathbf{T}$ be a consistent theory.

(1) If $\mathbf{T} \Longrightarrow \mathbf{T} \mathbf{h}_{\Pi_{n+1}}(\mathcal{N})$ then $\mathbf{T h}_{\Pi_{n+1}}(\mathbf{T})=\mathbf{T} \mathbf{h}_{\Pi_{n+1}}(\mathcal{N})$. 
(2) If $\mathbf{T} \mathbf{h}_{\Pi_{n+1}}(\mathcal{N})$ is recursively enumerable in $\mathbf{T}$, then $\mathbf{T} \mathbf{h}_{\Pi_{n+1}}(\mathcal{N})$ is recursive in $\mathbf{T}$.

Proof. (1): Let $\varphi \in \Pi_{n+1}$ such that $\mathbf{T} \vdash \varphi$ and $\mathfrak{A} \models \mathbf{T}$. Then $\mathfrak{A} \models \mathbf{T h}_{\Pi_{n+1}}(\mathcal{N})$; hence, $\mathcal{N} \prec_{n+1} \mathfrak{A}$. So, as $\mathfrak{A} \models \varphi, \mathcal{N} \models \varphi$.

(2): By the complementary theorem it is enough to prove that $\mathbf{T h}_{\Sigma_{n+1}}(\mathcal{N})$ is recursively enumerable in $\mathbf{T}$. We shall proceed by induction on $n \in \omega$.

$(n=0)$ : Trivial, $\mathbf{T h}_{\Sigma_{1}}(\mathcal{N})$ is recursively enumerable.

$(n \Longrightarrow n+1)$ : Since $\mathbf{T} \mathbf{h}_{\Sigma_{n+2}}(\mathcal{N})$ is a $\Sigma_{n+2}^{0}$ set and $\mathbf{T h}_{\Pi_{n+1}}(\mathcal{N})$ is a $\Pi_{n+1}^{0}$-complete set, the first one is recursively enumerable in the second one. By the induction hypothesis, $\mathbf{T h}_{\Pi_{n+1}}(\mathcal{N})$ is recursive in $\mathbf{T}$; so, $\mathbf{T} \mathbf{h}_{\Sigma_{n+2}}(\mathcal{N})$ is recursively enumerable in $\mathbf{T}$.

Lemma 4.2. Let $\mathbf{T}_{1}$ be a $\Sigma_{n+1}$-definable theory and let $\mathbf{T}_{2}$ be a $\Sigma_{n+1}$-axiomatized theory such that $\mathbf{T}_{1}+\mathbf{T}_{2}$ is consistent. Then $\mathbf{T}_{1}+\mathbf{T}_{2}$ does not imply $\mathbf{T h}_{\Pi_{n+1}}(\mathcal{N})$.

Proof. Assume that $\mathbf{T}_{1}+\mathbf{T}_{2} \Longrightarrow \mathbf{T h}_{\Pi_{n+1}}(\mathcal{N})$. Let $\mathfrak{A} \models \mathbf{T}_{1}+\mathbf{T}_{2}$. Then $\mathfrak{A} \models$ $\mathbf{T h}_{\Pi_{n+1}}(\mathcal{N})$; so, $\mathcal{N} \prec_{n+1} \mathfrak{A}$. Since $\mathbf{T}_{2}$ is $\Sigma_{n+1}$-axiomatized, $\mathbf{T h}_{\Pi_{n}}(\mathcal{N}) \Longrightarrow \mathbf{T}_{2}$; so, $\mathbf{T}_{1}+\mathbf{T h}_{\Pi_{n}}(\mathcal{N}) \Longrightarrow \mathbf{T h}_{\Pi_{n+1}}(\mathcal{N})$. Then, by Lemma $4.1, \mathbf{T h}_{\Pi_{n+1}}\left(\mathbf{T}_{1}+\mathbf{T h}_{\Pi_{n}}(\mathcal{N})\right)=$ $\mathbf{T h}_{\Pi_{n+1}}(\mathcal{N})$. Contradiction, the first set is $\Sigma_{n+1}^{0}$ and the second one is $\Pi_{n+1^{-}}^{0}$ complete.

4.1. Syntactical complexity. (A) Extensions of $\mathbf{I} \Sigma_{n+1}, \mathbf{B} \Sigma_{n+1}$ and $\mathbf{I} \Delta_{n+1}$

All these theories are $\Pi_{n+3}$-axiomatized; so, obviously, they have $\Pi_{n+3}-$ extensions. We shall see that for $n \geq 1$ this result is best possible; that is, they do not have $\Sigma_{n+3}-$ extensions. Next result extends a theorem of D. Leivant [16] who (essentially) proved, using uniform $\Sigma_{n+2}$-reflection in $\mathbf{I} \Sigma_{n+1}$, that $\mathbf{I} \Sigma_{n+1}$ does not have $\Sigma_{n+3^{-}}$ extensions.

Proposition 4.3. $\mathbf{B} \Sigma_{n+1}+\exp$ does not have $\Sigma_{n+3}$-extensions.

Proof. Assume that $\mathbf{B} \Sigma_{n+1}+\exp$ has a $\Sigma_{n+3^{-}}$extension, $\mathbf{T}$ say. Since $\mathbf{B} \Sigma_{n+1}+\exp$ is finitely axiomatized, there is $\varphi \in \Sigma_{n+3}$ such that $\mathbf{T} \vdash \varphi$ and $\varphi \Longrightarrow \mathbf{B} \Sigma_{n+1}+\mathbf{e x p}$. Let $\theta(x) \in \Pi_{n+2}^{-}$such that $\varphi \equiv \leq x \theta(x), \mathfrak{A}=\mathbf{T}$ and $a \in \mathfrak{A}$ such that $\mathfrak{A} \models \theta(a)$. Let $b \in \mathfrak{A}$ nonstandard and $p=\langle a, b\rangle$. Then $\mathcal{K}_{n+1}(\mathfrak{A}, p) \models \varphi ;$ so, $\mathcal{K}_{n+1}(\mathfrak{A}, p) \models \mathbf{B} \Sigma_{n+1}+$ exp. Which contradicts Theorem 3.5-(1).

Note that by the same argument as before, but taking the initial segment $\mathcal{I}_{n+1}(\mathfrak{A}, p)$ in place of $\mathcal{K}_{n+1}(\mathfrak{A}, p)$, it follows that there is no class of $\Sigma_{n+3}$-sentences, $\Gamma$, such that $\mathbf{B} \Sigma_{n+1}+\Gamma$ is a consistent extension of $\mathbf{I} \Sigma_{n+1}$.

\section{Theorem 4.4.}

(1) $\mathbf{I} \Sigma_{n+1}$ does not have $\Sigma_{n+3}$-extensions.

(2) $(n \geq 1) \mathbf{B} \Sigma_{n+1}$, and so $\mathbf{I} \Delta_{n+1}$, does not have $\Sigma_{n+3}$-extensions.

(3) $\mathbf{B} \Sigma_{1}$ and $\mathbf{I} \Delta_{1}$ do not have $\Sigma_{3}$-extensions consistent with exp. 
Proof. The results follow by Proposition 4.3, Slaman's Theorem and the facts that $\mathbf{I} \Sigma_{n+1} \Longrightarrow \mathbf{B} \Sigma_{n+1}+\exp$ and, for $n \geq 1, \mathbf{B} \Sigma_{n+1} \vdash \exp$.

To obtain optimal results, it only remains to find out if for extensions of $\mathbf{B} \Sigma_{1}$ and $\mathbf{I} \Delta_{1}$ the condition of being consistent with exp can be omitted.

Question 2. Do $\mathbf{B} \Sigma_{1}$ and $\mathbf{I} \Delta_{1}$ have $\Sigma_{3}$-extensions?

This question is related to Problem 3. In [20] it is proved that there exists a $\Pi_{1-}$ sentence, $\varphi$, provable in $\mathbf{I} \Delta_{0}+\exp$ but not in $\mathbf{I} \Delta_{0}$. Hence, it holds that $\mathbf{I} \Delta_{0}+\neg \varphi$ is a consistent extension of $\mathbf{I} \Delta_{0}+\neg \exp$. So, as $\mathbf{I} \Delta_{0}$ is $\Pi_{1}$-axiomatized, we have:

Corollary 4.5. If Problem 3 has an affirmative answer, then $\mathbf{B} \Sigma_{1}$ has recursively axiomatized $\cup_{1}$-extensions.

Therefore, optimal results for the case $n=0$ imply a negative answer to Problem 3 .

(B) Extensions of $\mathbf{I} \Sigma_{n+1}^{-}, \mathbf{B}_{\mathbf{s}} \Sigma_{n+1}^{-}, \mathbf{B} \Sigma_{n+1}^{-}$, and $\mathbf{U I} \Delta_{n+1}$

Fragment $\mathbf{I} \Sigma_{n+1}^{-}$is a sound $\bigvee_{n+2}$-axiomatized theory, so $\mathbf{T} \mathbf{h}_{\Pi_{n+2}}(\mathcal{N})$ is an extension of $\mathbf{I} \Sigma_{n+1}^{-}$; hence,

$$
\mathbf{T h}_{\Pi_{n+2}}(\mathcal{N}) \Longrightarrow \mathbf{I} \Sigma_{n+1}^{-} \Longrightarrow \mathbf{B}_{\mathbf{s}} \Sigma_{n+1}^{-} \Longrightarrow \mathbf{B} \Sigma_{n+1}^{-} \Longrightarrow \mathbf{U I} \Delta_{n+1} \Longrightarrow \mathbf{I} \Sigma_{n}
$$

Consequently, from Theorem 4.4-(1), we obtain the following result.

Theorem 4.6. $\mathbf{I} \Sigma_{n+1}^{-}, \mathbf{B}_{\mathbf{s}} \Sigma_{n+1}^{-}, \mathbf{B} \Sigma_{n+1}^{-}$and $\mathbf{U I} \Delta_{n+1}$ have $\Pi_{n+2}-$ extensions, and, for $n \geq 1$, they do not have $\Sigma_{n+2}$-extensions.

Let us see what happens in the second part of the above result for $n=0$. To this end, we first establish a result relating the syntactical complexity of a theory with the rate of growth of the recursive functions which are provably total in that theory. Proposition 4.7 below extends Claim 2.5.1 in [8] and generalizes theorems of R. Parikh and Bigorajska [5] on recursive functions provably total in $\mathbf{I} \Delta_{0}$ and $\mathbf{I} \Pi_{1}^{-}$, respectively.

Proposition 4.7. Let $\mathbf{T}$ be a theory and $\varphi(x, y) \in \Sigma_{1}$ such that $\mathbf{T}+\mathbf{B} \Sigma_{1} \vdash$ $\forall x \exists y \varphi(x, y)$.

(1) If $\mathbf{T}$ is $\Pi_{1}$-axiomatized, there is $m \in \omega$ such that

$$
\mathbf{T}+\mathbf{I} \Delta_{0} \vdash \forall x \exists y \leq(x+2)^{m} \varphi(x, y) .
$$

(2) If $\mathbf{T}$ is $\Sigma_{2}$-axiomatized, there is $m \in \omega$ such that

$$
\mathbf{T}+\mathbf{I} \Delta_{0} \vdash \exists z \forall x\left[z<x \rightarrow \exists y \leq(x+2)^{m} \varphi(x, y)\right] .
$$

Proof. (1): Assume that for all $m \in \omega, \mathbf{T}+\mathbf{I} \Delta_{0} \not \forall \forall x \exists y \leq(x+2)^{m} \varphi(x, y)$. Let c be a new constant and

$$
\mathbf{T}^{\prime}=\mathbf{T}+\mathbf{I} \Delta_{0}+\left\{\forall y \leq(\mathbf{c}+2)^{m} \neg \varphi(\mathbf{c}, y): m \in \omega\right\} .
$$


If $m_{1}<m_{2}$, then $\mathbf{I} \Delta_{0} \vdash(x+2)^{m_{1}}<(x+2)^{m_{2}}$; so, by compactness, there exists $\mathfrak{A} \models \mathbf{T}^{\prime}$. Let $\mathbf{d}$ be a new constant and $\mathbf{T}_{1}=\mathbf{E D}(\mathfrak{A})+\{a<\mathbf{d}: a \in \mathfrak{A}\}$ (where $\mathbf{E D}(\mathfrak{A})$ is the elementary diagram of $\mathfrak{A})$. By compactness, there exists $\mathfrak{B} \models \mathbf{T}_{1}$. Then $\mathfrak{B}$ is a proper elementary extension of $\mathfrak{A}$. Let $\mathfrak{C}$ be the substructure of $\mathfrak{B}$ with domain

$\left\{b \in \mathfrak{B}\right.$ : there exists $m \in \omega$ such that $\left.\mathfrak{B}=b<(\mathbf{c}+2)^{m}\right\}$.

Since $\mathfrak{C}$ is an initial segment in $\mathfrak{B}$ closed under function $(x+2)^{2}=y$, then it follows that $\mathfrak{B}$ is a proper $\Sigma_{0}$-elementary end extension of $\mathfrak{C}$. Hence, $\mathfrak{C}=\mathbf{T}+\mathbf{B} \Sigma_{1}$. So, $\mathfrak{C}=\forall x \exists y \varphi(x, y)$. Let $b \in \mathfrak{C}$ such that $\mathfrak{C}=\varphi(\mathbf{c}, b)$. Since $\varphi(x, y) \in \Sigma_{1}, \mathfrak{B}=\varphi(\mathbf{c}, b)$. Let $m \in \omega$ such that $b \leq(\mathbf{c}+2)^{m}$. Since $\mathfrak{A} \prec \mathfrak{B}, \mathfrak{A} \models \exists y \leq(\mathbf{c}+2)^{m} \varphi(\mathbf{c}, y)$. Contradiction, since $\mathfrak{A}=\mathbf{T}^{\prime}$.

(2): Since $\mathbf{T}$ is a $\Sigma_{2}$-axiomatized theory, there exists $\theta(u) \in \Pi_{1}^{-}$such that $\mathbf{T} \vdash$ $\exists u \theta(u)$ and $\mathbf{B} \Sigma_{1}+\exists u \theta(u) \vdash \forall x \exists y \varphi(x, y)$. Assume that for every $m \in \omega, \mathbf{T}+\mathbf{I} \Delta_{0} \not$ $\exists z \forall x\left[z<x \rightarrow \exists y \leq(x+2)^{m} \varphi(x, y)\right]$. Let $\mathbf{c}, \mathbf{d}$ be new constants and

$$
\mathbf{T}^{\prime}=\mathbf{I} \Delta_{0}+\theta(\mathbf{d})+\mathbf{d}<\mathbf{c}+\left\{\forall y \leq(\mathbf{c}+2)^{m} \neg \varphi(\mathbf{c}, y): m \in \omega\right\} .
$$

By compactness, there is $\mathfrak{A}=\mathbf{T}^{\prime}$. Let $\mathfrak{B}$ and $\mathfrak{C}$ as in the proof of part (1). Since $\mathfrak{C} \mid=\exists u \theta(u)$, reasoning as before we get the desired contradiction.

\section{Theorem 4.8.}

(1) $\mathbf{I} \Delta_{0}+\exp$ and $\mathbf{I} \Sigma_{1}^{-}$do not have $\Sigma_{2}$-extensions.

(2) $\mathbf{B}_{\mathbf{s}} \Sigma_{1}^{-}, \mathbf{B} \Sigma_{1}^{-}$and $\mathbf{U I} \Delta_{1}$ do not have $\Pi_{1}$-extensions; so, they do not have sound $\Sigma_{2}-$ extensions.

Proof. (1): Assume that $\mathbf{I} \Delta_{0}+\exp$ has a $\Sigma_{2}-$ extension, $\mathbf{T}$ say. Then, by Proposition 4.7, there exists $m \in \omega$ such that $\mathbf{T} \vdash \exists z \forall x>z\left(2^{x} \leq(x+2)^{m}\right)$. Which gives the desired contradiction.

(2): Since every $\Pi_{1}$-extension of $\mathbf{P}^{-}$is a sound theory, it is enough to show that $\mathbf{T} \mathbf{h}_{\Pi_{1}}(\mathcal{N})$ does not imply $\mathbf{U I} \Delta_{1}$. By Lemma 4.2 , there exists $\mathfrak{A} \models \mathbf{I} \Sigma_{1}+\mathbf{T h}_{\Pi_{1}}(\mathcal{N})$ such that $\mathfrak{A} \not \models \mathbf{T h}_{\Pi_{2}}(\mathcal{N})$. So, by Corollary 3.3 , there exists $p \in \mathcal{M}_{1}(\mathfrak{A})$ nonstandard. Since $\mathcal{K}_{1}(\mathfrak{A}, p) \prec_{1} \mathfrak{A}, \mathcal{K}_{1}(\mathfrak{A}, p) \models \mathbf{T h}_{\Pi_{1}}(\mathcal{N})+\mathbf{e x p}$. Towards a contradiction, assume that $\mathcal{K}_{1}(\mathfrak{A}, p)=\mathbf{U I} \Delta_{1}$. By the Claim in the proof of Theorem 3.5-(3), $\mathcal{K}_{2}\left(\mathcal{K}_{1}(\mathfrak{A}, p)\right)=\mathcal{K}_{1}(\mathfrak{A}, p)$, so by Lemma $3.4-(2 . b), \mathcal{K}_{1}(\mathfrak{A}, p) \models \mathbf{I} \Delta_{1}+$ exp. By Theorem 2.2, $\mathcal{K}_{1}(\mathfrak{A}, p) \models \mathbf{B} \Sigma_{1}+\mathbf{e x p}$, which contradicts Theorem 3.5.

Notice that part (1) of the above theorem follows from a result of Wilkie and Paris stating that $\mathbf{I} \Delta_{0}+\exp$ proves uniform $\Pi_{2}-$ reflection principle for $\mathbf{I} \Delta_{0}$ with respect to tableau provability (see [20]). Part (2) is essentially proved by Beklemishev in [4] (see the proof of corollary 4 there).

Nota 4.9. In [11] it is shown that, for each $n \geq 1$, there is a $\Pi_{n}$-formula, $y=\mathbb{K}_{n}(x)$, which satisfies the corresponding hierarchical versions of those properties of the formula $y=(x+2)^{2}$ necessary for the proof of Proposition 4.7, namely:

(a) $\mathbf{I} \Sigma_{n} \vdash \forall x \exists ! y\left(y=\mathbb{K}_{n}(x)\right)$; and 
(b) initial segments of $\mathfrak{B} \models \mathbf{I} \Sigma_{n}$ closed under function $y=\mathbb{K}_{n}(x)$ are $\Sigma_{n^{-}}$ elementary substructures of $\mathfrak{B}$.

Moreover, in [8] for each $n \geq 1$ it is presented a $\Pi_{n}$-formula, $y=\mathbb{K}_{n}^{z}(x)$, which expresses the iteration of the function $y=\mathbb{K}_{n}(x)$ and it is established that (see section 3 in [8] for details):

(c) $\mathbf{I} \Sigma_{n} \vdash z_{1}<z_{2} \rightarrow \mathbb{K}_{n}^{z_{1}}(x)<\mathbb{K}_{n}^{z_{2}}(x)$; and $\mathbf{I} \Sigma_{n} \vdash \forall x \exists$ !y $\left(y=\mathbb{K}_{n}^{m}(x)\right)$, for all $m \in \omega$.

Therefore, by repeating the arguments in the proof of Proposition 4.7, we obtain the following generalization of that result. Let us denote by $y=\mathbb{K}_{0}(x)$ the $\Delta_{0}$-formula $y=(x+2)^{2}$.

Proposition Let $\mathbf{T}$ be a theory and $\varphi(x, y) \in \Sigma_{n+1}$ such that $\mathbf{T}+\mathbf{B} \Sigma_{n+1} \vdash$ $\forall x \exists y \varphi(x, y)$.

1. If $\mathbf{T}$ is $\Pi_{n+1}$-axiomatized, there is $m \in \omega$ such that

$$
\mathbf{T}+\mathbf{I} \Sigma_{n} \vdash \forall x \exists y \leq \mathbb{K}_{n}^{m}(x) \varphi(x, y) .
$$

2. If $\mathbf{T}$ is $\Sigma_{n+2}$-axiomatized, there is $m \in \omega$ such that

$$
\mathbf{T}+\mathbf{I} \Sigma_{n} \vdash \exists z \forall x\left[z<x \rightarrow \exists y \leq \mathbb{K}_{n}^{m}(x) \varphi(x, y)\right] .
$$

In [8] it is also proved that $\mathbf{I} \Sigma_{n+1}^{-} \vdash \forall x \exists y\left(y=\mathbb{K}_{n}^{x+1}(x+2)\right)$. Hence, an argument similar to that of part (1) of Theorem 4.8 shows that there is no class of $\Sigma_{n+2^{-}}$ sentences, $\Gamma$, such that $\mathbf{B} \Sigma_{n+1}+\Gamma$ is a consistent extension of $\mathbf{I} \Sigma_{n+1}^{-}$. This fact can be also obtained from the results of [3]. There it is shown (see theorem 1) that $\mathbf{I} \Sigma_{n+1}^{-}$proves uniform $\Pi_{n+2}-$ reflection principle for $\mathbf{I} \Sigma_{n}$. Since $\mathbf{B} \Sigma_{n+1}$ is a $\Pi_{n+2}-$ conservative extension of $\mathbf{I} \Sigma_{n}, \mathbf{I} \Sigma_{n+1}^{-}$also proves $\Pi_{n+2}$-reflection principle for $\mathbf{B} \Sigma_{n+1}$ and, as a consequence, there is no class of $\Sigma_{n+2}$-sentences, $\Gamma$, such that $\mathbf{B} \Sigma_{n+1}+\Gamma$ is a consistent extension of $\mathbf{I} \Sigma_{n+1}^{-}$.

(C) Extensions of $\mathbf{I} \Pi_{n+1}^{-}, \mathbf{L} \Delta_{n+1}^{-}$and $\mathbf{I} \Delta_{n+1}^{-}$

All these theories are $\Sigma_{n+2}$-axiomatized; so, as they are sound, we have

$$
\mathbf{T h}_{\Pi_{n+1}}(\mathcal{N}) \Longrightarrow \mathbf{I} \Pi_{n+1}^{-} \Longrightarrow \mathbf{L} \Delta_{n+1}^{-} \Longrightarrow \mathbf{I} \Delta_{n+1}^{-} .
$$

Let us now consider the existence of $\Sigma_{n+1}$-extensions.

\section{Theorem 4.10.}

(1) $\mathbf{I} \Pi_{n+1}^{-}, \mathbf{L} \Delta_{n+1}^{-}$and $\mathbf{I} \Delta_{n+1}^{-}$have $\Pi_{n+1}$-extensions, and, for $n \geq 1$, they do not have $\Sigma_{n+1}$-extensions.

(2) $\mathbf{I \Pi}_{1}^{-}$does not have $\Sigma_{1}$-extensions.

(3) $\mathbf{I} \Delta_{1}^{-}$and $\mathbf{L} \Delta_{1}^{-}$do not have $\Sigma_{1}$-extensions consistent with exp.

Proof. (1): The results follow from Theorem 4.6 for $n>1$ and from Theorem 4.8 for $n=1$, since all these fragments imply $\mathbf{I} \Sigma_{n}^{-}$.

(2): Assume that there exists a $\Sigma_{1}$-extension of $\mathbf{I}_{1}^{-}, \mathbf{T}$ say. By Lemma 4.2, there exists $\mathfrak{A} \models \mathbf{T}$ such that $\mathfrak{A} \not \models \mathbf{T h}_{\Pi_{1}}(\mathcal{N})$ and, therefore, $\mathcal{K}_{1}(\mathfrak{A})$ is nonstandard. Since 
$\mathcal{K}_{1}(\mathfrak{A}) \prec_{1} \mathfrak{A}$ and $\mathbf{T}$ is a $\Sigma_{1}$-axiomatized theory, $\mathcal{K}_{1}(\mathfrak{A}) \models \mathbf{T}$; so, $\mathcal{K}_{1}(\mathfrak{A}) \models \mathbf{I} \Pi_{1}^{-}$. Then, from the fact that all elements of $\mathcal{K}_{1}(\mathfrak{A})$ are $\Sigma_{1}$-definable, we deduce that $\mathcal{K}_{1}(\mathfrak{A}) \models \mathbf{I} \Pi_{1}\left(\Longleftrightarrow \mathbf{I} \Sigma_{1}\right)$. In particular, $\mathcal{K}_{1}(\mathfrak{A}) \models \mathbf{B} \Sigma_{1}+$ exp. Which contradicts Theorem 3.5.

(3): Assume that there is a $\Sigma_{1}$-extension of $\mathbf{I} \Delta_{1}^{-}$consistent with $\exp , \mathbf{T}$ say. By Lemma 4.2 , there exists $\mathfrak{A} \models \mathbf{T}+\exp$ such that $\mathfrak{A} \not \models \mathbf{T h}_{\Pi_{1}}(\mathcal{N})$; so, $\mathcal{K}_{1}(\mathfrak{A})$ is nonstandard. Since $\mathbf{T}$ is $\Sigma_{1}$-axiomatized and $\mathcal{K}_{1}(\mathfrak{A}) \prec_{1} \mathfrak{A}, \mathcal{K}_{1}(\mathfrak{A}) \models \mathbf{T}+\mathbf{e x p}$; so, $\mathcal{K}_{1}(\mathfrak{A}) \models \mathbf{I} \Delta_{1}^{-}+$exp. Which contradicts Theorem 3.5.

4.2. Descriptive complexity. (A) $\Pi_{n+2}-$ extensions of $\mathbf{I} \Pi_{n+1}^{-}, \mathbf{L} \Delta_{n+1}^{-}$and $\mathbf{I} \Delta_{n+1}^{-}$ As we have noticed before, in [15] Kaye-Paris-Dimitracopoulos proved that for any $\Pi_{n+1}$ extension of $\mathbf{I} \Pi_{n+1}^{-}, \mathbf{T}$, it holds that $\mathbf{T} \Longleftrightarrow \mathbf{T h}_{\Pi_{n+1}}(\mathcal{N})$. Here we extend that result to any $\Pi_{n+2}$-extension. We also answer (partially) Problem 5.5 in [10].

Lemma 4.11. Let $\mathbf{T}$ be a $\Pi_{n+2}$-extension of $\mathbf{I} \Delta_{n+1}^{-}$such that for every $\mathfrak{A}=\mathbf{T}$, $\mathcal{K}_{n+1}(\mathfrak{A}) \models$ exp. Then $\mathbf{T h}_{\Pi_{n+1}}(\mathbf{T})=\mathbf{T h}_{\Pi_{n+1}}(\mathcal{N})$.

Proof. Assume that $\mathbf{T} \mathbf{h}_{\Pi_{n+1}}(\mathbf{T}) \neq \mathbf{T} \mathbf{h}_{\Pi_{n+1}}(\mathcal{N})$. By Lemma 4.1, $\mathbf{T}$ does not extend $\mathbf{T h}_{\Pi_{n+1}}(\mathcal{N})$; so, there is $\mathfrak{A} \models \mathbf{T}$ such that $\mathfrak{A} \not \models \mathbf{T h}_{\Pi_{n+1}}(\mathcal{N})$ and, therefore, $\mathcal{K}_{n+1}(\mathfrak{A})$ is nonstandard. Since $\mathbf{T}$ is $\Pi_{n+2}$-axiomatized and $\mathcal{K}_{n+1}(\mathfrak{A}) \prec_{n+1} \mathfrak{A}, \mathcal{K}_{n+1}(\mathfrak{A}) \models \mathbf{T}$. Hence, $\mathcal{K}_{n+1}(\mathfrak{A}) \models \mathbf{I} \Delta_{n+1}^{-}+$exp. Which contradicts Theorem 3.5.

\section{Theorem 4.12.}

(1) $\mathbf{L} \Delta_{n+1}^{-}+\exp$ and $\mathbf{I} \Delta_{n+1}^{-}+\exp$ are of type $n+2 \rightarrow n+1$.

(2) $\mathbf{I \Pi}_{n+1}^{-}$is of type $n+2 \rightarrow n+1$.

Proof. (1): Immediate from Lemma 4.11.

(2): Let $\mathbf{T}$ be a $\Pi_{n+2}$ extension of $\mathbf{I}_{n+1}^{-}$and $\mathfrak{A} \models \mathbf{T}$. Then $\mathcal{K}_{n+1}(\mathfrak{A}) \models \mathbf{T}$; so, $\mathcal{K}_{n+1}(\mathfrak{A}) \models \mathbf{I}_{n+1}^{-}$. Since all elements of $\mathcal{K}_{n+1}(\mathfrak{A})$ are $\Sigma_{n+1}$-definable, $\mathcal{K}_{n+1}(\mathfrak{A}) \models$ $\mathbf{I}_{n+1}$; so, $\mathcal{K}_{n+1}(\mathfrak{A}) \models$ exp. Hence, the result follows from Lemma 4.11.

The above result is best possible for $\mathbf{I} \Pi_{n+1}^{-}$and, when $n \geq 1$, also for $\mathbf{L} \Delta_{n+1}^{-}$and $\mathbf{I} \Delta_{n+1}^{-}$. However, it remains to eliminate the exponential function in the case $n=0$.

Question 3 . Are $\mathbf{L} \Delta_{1}^{-}$and $\mathbf{I} \Delta_{1}^{-}$of type $2 \rightarrow 1$, or $1 \rightarrow 1$ ?

Notice that this question is related to Problem 3. In fact, if Problem 3 has an affirmative answer, then, by Corollary $4.5, \mathbf{L} \Delta_{1}^{-}$has recursively axiomatized $\cup_{1}^{-}$ extensions; so, neither $\mathbf{L} \Delta_{1}^{-}$nor $\mathbf{I} \Delta_{1}^{-}$is of type $2 \rightarrow 1$. Nevertheless, we prove a weak version of previous question.

Proposition 4.13. $\mathbf{L} \Delta_{1}^{-}$and $\mathbf{I} \Delta_{1}^{-}$are of type $1 \stackrel{w}{\rightarrow} 1$. 
Proof. Let $\mathbf{T}$ be a $\Pi_{1}$-extension of $\mathbf{I} \Delta_{1}^{-}$. Then $\mathbf{T}$ is sound; so, $\mathbf{T}+\exp$ is a consistent $\Pi_{2}$-extension of $\mathbf{I} \Delta_{1}^{-}+\mathbf{e x p}$. By Theorem $4.12, \mathbf{T h}_{\Pi_{1}}(\mathbf{T}+\exp )=\mathbf{T h}_{\Pi_{1}}(\mathcal{N})$. This gives that $\mathbf{T h}_{\Pi_{1}}(\mathcal{N})$ is recursively enumerable in $\mathbf{T h}_{\Pi_{1}}(\mathbf{T}+\mathbf{e x p})$; so, also in $\mathbf{T} \mathbf{h}_{\Pi_{1}}(\mathbf{T})$. Hence, by Lemma $4.1, \mathbf{T} \mathbf{h}_{\Pi_{1}}(\mathcal{N})$ is recursive in $\mathbf{T} \mathbf{h}_{\Pi_{1}}(\mathbf{T})$. So, $\mathbf{I} \Delta_{1}^{-}$(and, consequently, also $\mathbf{L} \Delta_{1}^{-}$) is of type $1 \stackrel{w}{\longrightarrow} 1$, as required.

(B) $\Pi_{n+2}-$ extensions of $\mathbf{I} \Sigma_{n+1}^{-}, \mathbf{B}_{\mathbf{s}} \Sigma_{n+1}^{-}, \mathbf{B} \Sigma_{n+1}^{-}$and $\mathbf{U I} \Delta_{n+1}$ Since $\mathbf{T h}_{\Pi_{n+2}}(\mathcal{N}) \Longrightarrow \mathbf{I} \Sigma_{n+1}^{-} \Longrightarrow \mathbf{B}_{\mathbf{s}} \Sigma_{n+1}^{-}+\exp \Longrightarrow \mathbf{B} \Sigma_{n+1}^{-}+\exp \Longrightarrow \mathbf{U I} \Delta_{n+1}+$ $\exp \Longrightarrow \mathbf{I} \Delta_{n+1}^{-}+\exp$, then, by Theorem 4.12 , we have the following result.

Proposition 4.14. $\mathbf{I} \Sigma_{n+1}^{-}, \mathbf{B}_{\mathbf{s}} \Sigma_{n+1}^{-}+\exp , \mathbf{B} \Sigma_{n+1}^{-}+\exp$ and $\mathbf{U I} \Delta_{n+1}+\exp$ are of type $n+2 \rightarrow n+1$.

Now we shall improve this result: we shall see that $\mathbf{I} \Sigma_{n+1}^{-}$is of type $n+2 \rightarrow n+2$ and a weaker version for $\mathbf{B} \Sigma_{n+1}^{-}+\exp$ and $\mathbf{U I} \Delta_{n+1}+\exp$, namely, these theories are of type $n+2 \stackrel{w}{\longrightarrow} n+2$.

Lemma 4.15. Let $\mathbf{T}$ be $\Pi_{n+2}$-axiomatized and $\varphi$ a $\Sigma_{n+2}$-sentence such that $\mathbf{I} \Sigma_{n+1}^{-}+$ $\mathbf{T}+\varphi$ is consistent.

(1) If $\mathbf{T}+\varphi \Longrightarrow \mathbf{B} \Sigma_{n+1}^{-}$, then $\mathbf{I} \Sigma_{n+1}^{-}+\mathbf{T}+\varphi \Longleftrightarrow \mathbf{T h}_{\Pi_{n+2}}(\mathcal{N})$.

(2) If $\mathbf{I} \Sigma_{n}+\mathbf{I} \Pi_{n+1}^{-}+\mathbf{T}+\varphi \Longrightarrow \mathbf{B} \Sigma_{n+1}^{-}$, then $\mathbf{I} \Sigma_{n+1}^{-}+\mathbf{T}+\varphi \Longleftrightarrow \mathbf{T h}_{\Pi_{n+2}}(\mathcal{N})$.

Proof. Even though it is enough to prove part 2, we shall prove both parts. Let $\theta(x)$ be a $\Pi_{n+1}^{-}$formula such that $\varphi \equiv \exists x \theta(x)$.

$(\Longrightarrow)$ : Assume that there is $\mathfrak{A} \models \mathbf{I} \Sigma_{n+1}^{-}+\mathbf{T}+\varphi$ such that $\mathfrak{A} \not \mathbf{T h}_{\Pi_{n+2}}(\mathcal{N})$. Since $\mathfrak{A}=\mathbf{L} \Pi_{n+1}^{-}\left(\Longleftrightarrow \mathbf{I} \Sigma_{n+1}^{-}\right)$, there exists $a \in \mathcal{M}_{n+1}(\mathfrak{A})$ such that $\mathfrak{A} \models a=(\mu x)(\theta(x))$.

(1): By Corollary 3.3, there exists $b \in \mathcal{M}_{n+1}(\mathfrak{A})$ nonstandard. Take $p=\langle a, b\rangle$. Since $\mathcal{K}_{n+1}(\mathfrak{A}, p) \models \mathbf{T}+\varphi$, by hypothesis, $\mathcal{K}_{n+1}(\mathfrak{A}, p) \models \mathbf{B} \Sigma_{n+1}^{-}$. Which contradicts Theorem 3.5 since $p \in \mathcal{M}_{n+1}(\mathfrak{A})$.

(2): By Corollary 3.7, there exists $b \in \mathcal{D}_{n+1}(\mathfrak{A})$ nonstandard such that $\mathcal{K}_{n+1}(\mathfrak{A}, a, b) \models$ $\mathbf{I}_{n+1}^{-}$. Let $p=\langle a, b\rangle$. Then $\mathcal{K}_{n+1}(\mathfrak{A}, p) \models \mathbf{I} \Sigma_{n}+\mathbf{I}_{n+1}^{-}+\mathbf{T}+\varphi$; so, by hypothesis, $\mathcal{K}_{n+1}(\mathfrak{A}, p) \models \mathbf{B} \Sigma_{n+1}^{-}$. Which contradicts Theorem 3.5 since $p \in \mathcal{M}_{n+1}(\mathfrak{A})$.

$(\Longleftarrow)$ : It is enough to prove that $\mathbf{T}+\varphi$ is a sound theory. Let $\mathfrak{A}$ be a model of $\mathbf{I} \Sigma_{n+1}^{-}+\mathbf{T}+\varphi$. By part $\Longrightarrow$, it holds that $\mathfrak{A} \models \mathbf{T h}_{\Pi_{n+2}}(\mathcal{N})$; hence, $\mathcal{N} \prec_{n+2} \mathfrak{A}$. So, $\mathcal{N} \models \mathbf{T}+\varphi$.

Theorem 4.16.

(1) $\mathbf{I} \Sigma_{n+1}^{-}$is of type $n+2 \rightarrow n+2$.

(2) $\mathbf{B} \Sigma_{n+1}^{-}+\exp$ (and, consequently, also $\mathbf{B}_{\mathbf{s}} \Sigma_{n+1}^{-}+\mathbf{e x p}$ ) is of type $n+2 \stackrel{w}{\rightarrow} n+2$.

Proof. (1): Immediate from Lemma 4.15.

(2): Let $\mathbf{T}$ be a $\Pi_{n+2}$ extension of $\mathbf{B} \Sigma_{n+1}^{-}+\exp$. Then $\mathbf{T} \Longrightarrow \mathbf{L} \Delta_{n+1}^{-}+\mathbf{e x p}$. By Theorem 4.12, $\mathbf{T h}_{\Pi_{n+1}}(\mathbf{T})=\mathbf{T} \mathbf{h}_{\Pi_{n+1}}(\mathcal{N})$ and $\mathcal{N} \models \mathbf{T}$; so, $\mathbf{I} \Sigma_{n+1}^{-}+\mathbf{T}$ is consistent. 
By Lemma 4.15, $\mathbf{T h}_{\Pi_{n+2}}\left(\mathbf{I} \Sigma_{n+1}^{-}+\mathbf{T}\right)=\mathbf{T h}_{\Pi_{n+2}}(\mathcal{N})$. Then $\mathbf{T h}_{\Pi_{n+2}}(\mathcal{N})$ is recursively enumerable in $\mathbf{T h}_{\Pi_{n+2}}(\mathbf{T})$. Hence, by Lemma $4.1, \mathbf{T h}_{\Pi_{n+2}}(\mathcal{N})$ is recursive in $\mathbf{T h}_{\Pi_{n+2}}(\mathbf{T})$.

Our result on descriptive complexity of extensions of $\mathbf{I} \Sigma_{n+1}^{-}$is best possible; however, the following questions are left unanswered for the collection scheme.

\section{Question 4.}

(1) Is $\mathbf{B} \Sigma_{n+1}^{-}+\exp$ of type $n+2 \rightarrow n+2$ ?

(2) Is $\mathbf{B} \Sigma_{1}^{-}$of type $2 \rightarrow 2$, or $2 \stackrel{w}{\rightarrow} 2$, or $2 \rightarrow 1$, or $2 \stackrel{w}{\rightarrow} 1$ ?

Part (2) is connected with Problem 3. Note that by Corollary 4.5, if Problem 3 has an affirmative answer, then $\mathbf{B} \Sigma_{1}^{-}$has recursively axiomatized $\cup_{1}$-extensions; so, it is not of type $2 \stackrel{w}{\longrightarrow} 1$. We shall see now that part (1) is related to the existence of nonstandard $\Pi_{n+1}$-definable elements in models of $\mathbf{B} \Sigma_{n+1}$.

Proposition 4.17. If Question 1 has an affirmative answer, then $\mathbf{B} \Sigma_{n+1}^{-}+\mathbf{e x p}$ is of type $n+2 \rightarrow n+2$.

Proof. Let $\mathbf{T}$ be a $\Pi_{n+2}$-extension of $\mathbf{B} \Sigma_{n+1}^{-}+\mathbf{e x p}$. Let us see that $\mathbf{T} \Longrightarrow \mathbf{T h}_{\Pi_{n+2}}(\mathcal{N})$. Since $\mathbf{B} \Sigma_{n+1}$ is a $\Sigma_{n+3^{-}}$conservative extension of $\mathbf{B} \Sigma_{n+1}^{-}$(see Theorem 2.4 in [15]), it suffices to prove that $\mathbf{T}+\mathbf{B} \Sigma_{n+1} \Longrightarrow \mathbf{T} \mathbf{h}_{\Pi_{n+2}}(\mathcal{N})$. Assume that there exists $\mathfrak{A} \models \mathbf{T}+\mathbf{B} \Sigma_{n+1}$ such that $\mathfrak{A} \not \mathbf{T h}_{\Pi_{n+2}}(\mathcal{N})$. By the hypothesis, there is $p \in \mathcal{D}_{n+1}(\mathfrak{A})$ nonstandard. Since $\mathbf{T}$ is $\Pi_{n+2}$-axiomatized, $\mathcal{K}_{n+1}(\mathfrak{A}, p) \models \mathbf{T}$; so, $\mathcal{K}_{n+1}(\mathfrak{A}, p) \models \mathbf{B} \Sigma_{n+1}^{-}+$exp. Which contradicts Theorem 3.5.

For the uniform $\Delta_{n+1}$-induction scheme, we have obtained that $\mathbf{U I} \Delta_{n+1}+\exp$ is of type $n+2 \rightarrow n+1$ from the fact that this fragment is an extension of $\mathbf{I} \Delta_{n+1}^{-}+\mathbf{e x p}$. Since $\mathbf{U I} \Delta_{n+1}+\exp$ and $\mathbf{B} \Sigma_{n+1}^{-}+\exp$ are equivalent (see Theorem 2.4), it is immediate that $\mathbf{U I} \Delta_{n+1}+\exp$ is of type $n+2 \stackrel{w}{\rightarrow} n+2$. However, by Lemma $3.4-(2 . b)$, we can apply the same reasoning as in Lemma 4.15 and Theorem 4.16 to show, independently of the equivalence between $\mathbf{U I} \Delta_{n+1}+\exp$ and $\mathbf{B} \Sigma_{n+1}^{-}+\exp$, that

Proposition 4.18. UI $\Delta_{n+1}+\exp$ is of type $n+2 \stackrel{w}{\longrightarrow} n+2$.

\section{On $\Delta_{n+1}$-schemes}

In this section, we make use of results in the previous section in order to determine quantifier complexity, (non)finite axiomatizability and relative strength of the fragments studied in this work. We focus on fragments for $\Delta_{n+1}$-formulas and the scheme $\mathbf{B}_{\mathbf{s}} \Sigma_{n+1}^{-}$since the properties of these theories are not well known. First we state general conditions for a theory $\mathbf{T}$ to establish its axiomatization properties. 
Lemma 5.1. If $\mathbf{T} \mathbf{h}_{\Pi_{n+2}}(\mathcal{N}) \Longrightarrow \mathbf{T} \Longrightarrow \mathbf{I} \Delta_{n+1}^{-}$, then $\mathbf{T}$ is not finitely axiomatized.

Proof. Assume $\mathbf{T}$ is finitely axiomatized. Then there exists $\varphi \in \mathbf{T h}_{\Pi_{n+2}}(\mathcal{N})$ such that $\varphi \Longrightarrow \mathbf{T}$ and hence $\varphi+\exp \Longrightarrow \mathbf{I} \Delta_{n+1}^{-}+\exp$. Since $\mathbf{I} \Delta_{n+1}^{-}+\exp$ is of type $n+$ $2 \rightarrow n+1$ (Theorem 4.12), it holds that $\varphi+\exp \Longrightarrow \mathbf{T h}_{\Pi_{n+1}}(\mathcal{N})$. Contradiction.

Lemma 5.2. Let $\mathbf{T}$ be a theory consistent with exp.

(1) If $\mathbf{T}$ is $\Sigma_{n+1}$-definable and extends $\mathbf{I} \Delta_{n+1}^{-}$, then $\mathbf{T}$ is not $\Pi_{n+2}$-axiomatized.

(2) If $\mathbf{T}$ is a sound $\Sigma_{n+2}$-definable extension of $\mathbf{U I} \Delta_{n+1}$, then $\mathbf{T}$ is not $\cup_{n+2^{-}}$ axiomatized.

Proof. (1): Assume $\mathbf{T}$ is $\Pi_{n+2}$-axiomatized. Then $\mathbf{T}+\exp$ is a $\Pi_{n+2}-$ extension of $\mathbf{I} \Delta_{n+1}^{-}+\exp$ and hence, by Theorem 4.12 , it follows that $\mathbf{T}+\exp \Longrightarrow \mathbf{T h}_{\Pi_{n+1}}(\mathcal{N})$. Which is impossible since $\mathbf{T}$ is $\Sigma_{n+1}$-definable.

(2): Assume that $\mathbf{T} \Longleftrightarrow \mathbf{T}_{1}+\mathbf{T}_{2}$ where $\mathbf{T}_{1}$ is $\Sigma_{n+2^{-}}$axiomatized and $\mathbf{T}_{2}$ is $\Pi_{n+2^{-}}$ axiomatized. Since $\mathbf{T}_{1}$ is sound, $\mathbf{T h}_{\Pi_{n+1}}(\mathcal{N}) \Longrightarrow \mathbf{T}_{1}$. Let $\mathbf{T}_{3}=\mathbf{T}+\mathbf{I} \Sigma_{n+1}+$ $\mathbf{T h}_{\Pi_{n+1}}(\mathcal{N})$. Since $\mathbf{T}_{3}$ is consistent and $\Sigma_{n+2}$-definable, $\mathbf{T}_{3} \nLeftarrow \mathbf{T h}_{\Pi_{n+2}}(\mathcal{N})$; so, there exists $\mathfrak{A} \models \mathbf{T}_{3}$ such that $\mathfrak{A} \not \models \mathbf{T h}_{\Pi_{n+2}}(\mathcal{N})$. By Corollary 3.3, there exists $p \in \mathcal{M}_{n+1}(\mathfrak{A})$ nonstandard. Then $\mathcal{K}_{n+1}(\mathfrak{A}, p) \models \mathbf{T h}_{\Pi_{n+1}}(\mathcal{N})+\mathbf{T}_{2}$ and; hence, $\mathcal{K}_{n+1}(\mathfrak{A}, p)=\mathbf{U I} \Delta_{n+1}+$ exp. By the Claim in the proof of Theorem 3.5-(3), $\mathcal{K}_{n+2}\left(\mathcal{K}_{n+1}(\mathfrak{A}, p)\right)=\mathcal{K}_{n+1}(\mathfrak{A}, p)$, so by Lemma 3.4-(2.b), $\mathcal{K}_{n+1}(\mathfrak{A}, p) \models \mathbf{I} \Delta_{n+1}$. By Theorem 2.2, $\mathcal{K}_{n+1}(\mathfrak{A}, p)=\mathbf{B} \Sigma_{n+1}+$ exp. Which contradicts Theorem 3.5.

Now we apply both lemmas to obtain the basic information on the quantifier complexity of the considered $\Delta_{n+1}$-schemes and fragment $\mathbf{B}_{\mathbf{s}} \Sigma_{n+1}^{-}$. First, let us observe that all these fragments are recursively axiomatized and, consequently, $\Sigma_{1}-$ definable.

\section{Theorem 5.3.}

(1) $\mathbf{L} \Delta_{n+1}^{-}, \mathbf{I} \Delta_{n+1}^{-}, \mathbf{U I} \Delta_{n+1}$ and $\mathbf{B}_{\mathbf{s}} \Sigma_{n+1}^{-}$are not finitely axiomatized.

(2) $\mathbf{L} \Delta_{n+1}^{-}$and $\mathbf{I} \Delta_{n+1}^{-}$are not $\Pi_{n+2}$-axiomatized.

(3) $\mathbf{B}_{\mathbf{s}} \Sigma_{n+1}^{-}$and $\mathbf{U I} \Delta_{n+1}$ are not $\cup_{n+2}$-axiomatized.

Finally, from the obtained results, we can deduce the following properties on the relative strength of the considered fragments.

\section{Theorem 5.4 .}

(1) $\mathbf{I} \Delta_{n+1} \longmapsto \mathbf{U I} \Delta_{n+1} \longmapsto \mathbf{I} \Delta_{n+1}^{-} \models \mathbf{I} \Sigma_{n}$.

(2) $\mathbf{L} \Delta_{n+1}^{-}$does not imply $\mathbf{U I} \Delta_{n+1}$, and $\mathbf{U L} \Delta_{n+1}$ does not imply $\mathbf{I} \Delta_{n+1}$.

(3) (Answer to problem 4 in [4]) There is no recursively enumerable set of true $\Pi_{n+2}$-sentences which extends $\mathbf{I} \Delta_{n+1}^{-}$. In particular, $\mathbf{I} \Delta_{0}+\exp$ does not extend $\mathbf{I} \Delta_{1}^{-}$. 
Proof. By Theorem 5.3-(2) and the fact that $\mathbf{I} \Sigma_{n}$ is $\Pi_{n+2}$-axiomatized, it follows that $\mathbf{I} \Sigma_{n}$ does not imply $\mathbf{I} \Delta_{n+1}^{-}$. By Lemma $5.2-(3)$ and the fact that $\mathbf{I} \Delta_{n+1}^{-}$and $\mathbf{L} \Delta_{n+1}^{-}$are $\Sigma_{n+2^{-}}$axiomatized, it follows that neither $\mathbf{I} \Delta_{n+1}^{-}$nor $\mathbf{L} \Delta_{n+1}^{-}$implies $\mathbf{U I} \Delta_{n+1}$. By Theorem 4.4, it follows that $\mathbf{I} \Delta_{n+1}+\exp$ does not have $\Sigma_{n+3^{-}}$ extensions; so, neither $\mathbf{U I} \Delta_{n+1}$ nor $\mathbf{U L} \Delta_{n+1}$ implies $\mathbf{I} \Delta_{n+1}$ since $\mathbf{U I} \Delta_{n+1}$ and $\mathbf{U L} \Delta_{n+1}$ are $\bigvee_{n+2}$-axiomatized. Finally, observe that part (3) follows by the fact that $\mathbf{I} \Delta_{n+1}^{-}+\exp$ is of type $n+2 \rightarrow n+1$.

Concerning to the scheme $\mathbf{B}_{\mathbf{s}} \Sigma_{n+1}^{-}$the following question on its quantifier complexity remains unanswered.

Question 5. Is $\mathbf{B}_{\mathbf{s}} \Sigma_{n+1}^{-}$a $\bigvee_{n+2}$-axiomatized theory?

Using the same reasoning as for its parameter counterpart, it is easy to check that $\mathbf{B}_{\mathbf{s}} \Sigma_{n+1}^{-}$is a $\Pi_{n+3}$-axiomatized theory. However, we do not even know if it is a $\Sigma_{n+3}$-axiomatized theory. In fact, we have the following result.

Proposition 5.5. The following conditions are equivalent.

(1) $\mathbf{B} \Sigma_{n+1}^{-} \Longleftrightarrow \mathbf{B}_{\mathbf{s}} \Sigma_{n+1}^{-}$.

(2) $\mathbf{B}_{\mathbf{s}} \Sigma_{n+1}^{-}$is $\bigvee_{n+2}$-axiomatized.

(3) $\mathbf{B}_{\mathbf{s}} \Sigma_{n+1}^{-}$is $\Sigma_{n+3}$-axiomatized.

Proof. Since $\mathbf{B} \Sigma_{n+1}^{-}$is $\bigvee_{n+2}$ axiomatized, (1) $\Longrightarrow(2)$ is immediate. The implication $(2) \Longrightarrow(3)$ is trivial and (3) $\Longrightarrow$ (1) follows from the fact that $\mathbf{B} \Sigma_{n+1}$ is $\Sigma_{n+3^{-}}$ conservative over $\mathbf{B} \Sigma_{n+1}^{-}$.

\section{References}

[1] Adamovicz, Z.; Bigorajska, T. Functions provably total in $I^{-} \Sigma_{1}$. Fundamenta Mathematicae, 132:189-194, 1989.

[2] Beklemishev, L.D. Induction rules, reflection principles and provably recursive functions. Annals of Pure and Applied Logic, 85(3):193-242, 1997.

[3] Beklemishev, L.D. Parameter free induction and provably total computable functions. Theoretical Computer Science, 224(1-2):13-33, 1999.

[4] Beklemishev, L.D. On the induction schema for decidable predicates. The Journal of Symbolic Logic, 68(1):17-34, 2003.

[5] Bigorajska, T. On $\Sigma_{1}$-definable functions provably total in $I \Pi_{1}^{-}$. Mathematical Logic Quartely, 41:135-137, 1995.

[6] Clote, P; Krajíček, J. Open Problems. In Arithmetic, Proof Theory and Computational Complexity, 1-19. Oxford Logic Guides, 23. Oxford University Press, Oxford, 1993.

[7] Cordón Franco, A. Extensiones de Fragmentos de la Aritmética. Ph.D. Thesis. Universidad de Sevilla, 2003.

[8] Cordón Franco, A; Fernández Margarit, A.; Lara Martín, F.F. On the quantifier complexity of $\Delta_{n+1}(\mathbf{T})-$ induction. Archive for Mathematical Logic, 43(3):371-398, 2004.

[9] Cordón Franco, A; Fernández Margarit, A.; Lara Martín, F.F. Fragments of Arithmetic on $\Delta_{n+1}$-formulas. Preprint, Sevilla, December 2004. 
[10] Fernández Margarit, A.; Lara Martín, F.F. Some Results on $\mathbf{L} \Delta_{n+1}^{-}$. Mathematical Logic Quarterly, 47(4):503-512, 2001.

[11] Fernández Margarit, A.; Lara Martín, F.F. Induction, Minimization and Collection for $\Delta_{n+1}(\mathbf{T})$-formulas. Archive for Mathematical Logic, 43(4):505-541, 2004.

[12] Hájek, P.; Pudlák, P. Metamathematics of First-Order Arithmetic. Perspectives in Mathematical Logic, Springer Verlag, 1993.

[13] Kaye, R. Diophantine and Parameter-free Induction. Ph.D. Thesis. University of Manchester, 1987.

[14] Kaye, R. Models of Peano Arithmetic. Oxford Logic Guides, 15. Clarendon Press. Oxford, 1991.

[15] Kaye, R.; Paris, J; Dimitracopoulos, C. On parameter free induction schemas. The Journal of Symbolic Logic, 53(4):1082-1097, 1988.

[16] Leivant, D. The optimality of induction as an axiomatization of arithmetic. The Journal of Symbolic Logic. 48(1):182-184, 1983.

[17] Paris, J.; Kirby, L. $\Sigma_{n}$-collection schemas in arithmetic. Logic Colloquium'77, 199-209. North Holland, 1978.

[18] Slaman, T. $\Sigma_{n}-$ Bounding and $\Delta_{n}$-induction. Proceedings of the American Mathematical Society, 132(8):2449-2456, 2004.

[19] Wilkie, A. Some Results and Problems on Weak Systems of Arithmetic. Logic Colloquium'77, 285-296. North Holland, 1978.

[20] Wilkie, A; Paris, J. On the scheme of induction for bounded arithmetic formulas. Annals of Pure and Applied Logic, 35(3):261-302, 1987.

[21] Wilkie, A; Paris, J. On the existence of end extensions of models of bounded induction. Logic, Methodology and Philosophy of Science VIII, 143-161. North-Holland, Amsterdam 1989. 